DESY 07-198

hep-ph/0711.2196

\title{
Baryon scattering at high energies: wave function, impact factor, and gluon radiation
}

\author{
J. Bartels $^{a) *}$ and L. Motyka ${ }^{a), b) \dagger}$ \\ a) II Institute for Theoretical Physics, University of Hamburg, Germany \\ b) Institute of Physics, Jagellonian University, Kraków, Poland
}

November 14, 2007

\begin{abstract}
The scattering of a baryon consisting of three massive quarks is investigated in the high energy limit of perturbative QCD. A model of a relativistic proton-like wave function, dependent on valence quark longitudinal and transverse momenta and on quark helicities, is proposed, and we derive the baryon impact factors for two, three and four $t$-channel gluons. We find that the baryonic impact factor can be written as a sum of three pieces: in the first one a subsystem consisting of two of the three quarks behaves very much like the quark-antiquark pair in $\gamma^{*}$ scattering, whereas the third quark acts as a spectator. The second term belongs to the odderon, whereas in the third ( $C$-even) piece all three quarks participate in the scattering. This term is new and has no analogue in $\gamma^{*}$ scattering. We also study the small $x$ evolution of gluon radiation for each of these three terms. The first term follows the same pattern of gluon radiation as the $\gamma^{*}$-initiated quark-antiquark dipole, and, in particular, it contains the BFKL evolution followed by the $2 \rightarrow 4$ transition vertex (triple Pomeron vertex). The odderon-term is described by the standard BKP evolution, and the baryon couples to both known odderon solutions, the Janik-Wosiek solution and the BLV solution. Finally, the $t$-channel evolution of the third term starts with a three reggeized gluon state which then, via a new $3 \rightarrow 4$ transition vertex, couples to the four gluon (two-Pomeron) state. We briefly discuss a few consequences of these findings, in particular the pattern of unitarization of high energy baryon scattering amplitudes.
\end{abstract}

*Email: bartels@mail.desy.de

${ }^{\dagger}$ E-mail: motyka@th.if.uj.edu.pl 


\section{Introduction}

In recent years deep inelastic electron proton or electron nucleus scattering (DIS) at small $x$ has attracted much interest, and it has stimulated intense studies of high energy QCD. At high energies, the total cross section of a virtual photon scattering on a target, in a first approximation, can be described in terms of a photon impact factor and a Balitsky-Fadin-Kuraev-Lipatov (BFKL) Green's function [1-3]. When restricting to the large $N_{c}$ limit, and assuming a large target, unitarity corrections to this first approximation are described by the nonlinear Balitsky-Kovchegov (BK) equation $[4,5]$ which, in the language of BFKL Green's functions, represents the infinite sum of fan diagrams [6]. The BK equation was initially obtained in the $s$-channel color dipole picture (in the large $N_{c}$ limit) $[7,8]$. Beyond the large $N_{c}$ limit one has to include the full color structure of the $2 \rightarrow 4$ reggeized gluon vertex [9] which leads to the Balitsky hierarchy of integral equations [4] or to the Jalilian-Marian-Iancu-McLerran-Weigert-Leonidov-Kovner (JIMWLK) equations [10]. In many of these calculations the incoming virtual photon plays a vital rôle: its large virtuality $Q^{2}$ justifies the use of perturbation theory, and its impact factor consists of a quark-antiquark pair which forms a color dipole configuration. This simple structure is also intimately connected with the fan-like structure of the diagrams resumed by the nonlinear BK equation.

The advent of the LHC challenges us with the task of developing a theoretical understanding of scattering in high energy proton-proton collisions, which is related to the structure of unitarity corrections in baryonbaryon scattering. In this paper we will perform a study of the high energy behavior of baryon scattering within perturbative QCD. It is clear that the problem of high energy nucleon scattering is much more complex than it was in the virtual photon case. First of all, in nucleon-nucleon or nucleon-nucleus scattering the incoming projectiles are nonperturbative, and the accuracy of perturbative calculations is not under good theoretical control. We shall circumvent this problem by studying a fictitious scattering process of a heavy and small baryonium system, in analogy to the heavy onium proposed as a test case for perturbative unitarity corrections in DIS [8]. For such processes the perturbative calculations provide reliable results. Next, the baryonium scattering is expected to differ significantly from the onium scattering. The main reason is the difference of the color structure: in contrast to the color dipole the baryon is a color singlet formed by three valence quarks. Also, the application of the large $N_{c}$ limit which played the crucial rôle in the construction of the dipole model is rather difficult in the baryon case: one needs exactly $N_{c}$ quarks to build the color singlet of the $S U\left(N_{c}\right)$ group, and this system becomes rather complex for $N_{c} \rightarrow \infty$. In fact, a few years ago, it was explicitly pointed out [11] that the simple picture of gluon radiation which has emerged in the QCD dipole picture does not work in the case of an incoming three quark color singlet system; however, no alternative solution had been derived. Thus, we shall address the issue of gluon radiation from three quarks at $N_{c}=3$, within a perturbative baryonic system and compare with the perturbative quark-antiquark system.

The basic and universal object that characterizes properties of the baryon is its wave function. Inspired by the success of the concept of the photon wave function [7] which turned out to be very fruitful in studies of high energy scattering, we start from a local three-fermion quark current operator with the quantum numbers of the proton and construct a relativistic invariant infinite momentum frame wave function for the lowest 
Fock component of the baryon, consisting of three valence quarks. The resulting wave function contains a non-trivial dependence on quark helicities and angular momenta. For the current operator we chose the baryonic operator proposed by Ioffe [12], which has been shown to provide a reasonable phenomenological prescription of the nucleon properties [13]. In order to take into account the nonperturbative nature of the baryon we make use of the Borel transform technique which has been developed in the context of QCD sum rules.

This paper is not intended yet to deal with a detailed phenomenology of the baryon structure and scattering, - thus we do not attempt, for example, to tune the obtained wave function to describe the existing data on proton form-factors and high energy scattering. Nevertheless, apart from developing a theoretical laboratory for studying scattering of baryon states at high energies, one may hope that our perturbative analysis finds structures which remain also relevant beyond the perturbatively safe region. An extrapolation of our results on the heavy baryonium to the realistic proton case may, therefore, very well allow for some useful phenomenology. More detailed studies in this direction will be left for future work.

Starting from integrals over squares of these baryonic wave functions and coupling $t$-channel gluons to the three quark lines we define baryonic impact factors, in close analogy with the photon impact factor in deep inelastic electron proton scattering. The small- $x$ evolution of baryon scattering amplitude will be analyzed, again, following the strategy developed in the context of the virtual photon scattering $[9,14,15]$. First we consider, in lowest order, the elastic scattering of the baryonic system on a single quark: by coupling two $t$-channel gluons to the three-quark system, the baryonic impact factor is obtained. Three or four $t$-channel gluons appear if one considers, again at lowest order, multi-particle amplitudes, e.g. $3 \rightarrow 3$ processes in a suitably defined high energy limit. In the next step, one considers higher order diagrams in the leading logarithmic approximation: this leads to rapidity evolution equations, describing the radiation of gluons from the three-quark system.

Our main results are the following. We propose a model of the baryon wave function with a non-trivial quark helicity and angular momentum structure. Then we express the baryon impact factor in terms of the wave function, for an arbitrary number of coupling gluons. The obtained baryonic impact factor can be written as a sum of several pieces, each of them having its own evolution equation. First, there is a term in which one pair out of the three quarks scatters whereas the third quark acts as a spectator. Although the two quarks which participate in the interaction are in a color anti-triplet configuration, they behave very much like the quark-antiquark pair in the photon case. In the lowest order, two $t$-channel gluons couple to this quark pair. In higher order the two gluons start to reggeize and to produce the full BFKL ladder, while the third quark of the baryon state remains an inactive spectator. Also, the well-known $2 \rightarrow 4$ gluon vertex appears, indicating the beginning of the same fan-like structure as in the quark-antiquark case. Altogether, this piece of the baryon impact factor radiates gluons in very much the same way as the quark-antiquark pair in the photon case.

Next, there is the odderon term, similar to the one discussed in [16]: here all three quarks participate, and the $t$ channel state carries $C=-$. In lowest order, three gluons couple to the three quarks; in higher order the state evolves according to the Bartels-Kwieciński-Praszałowicz (BKP) evolution equation [17, 18]. 

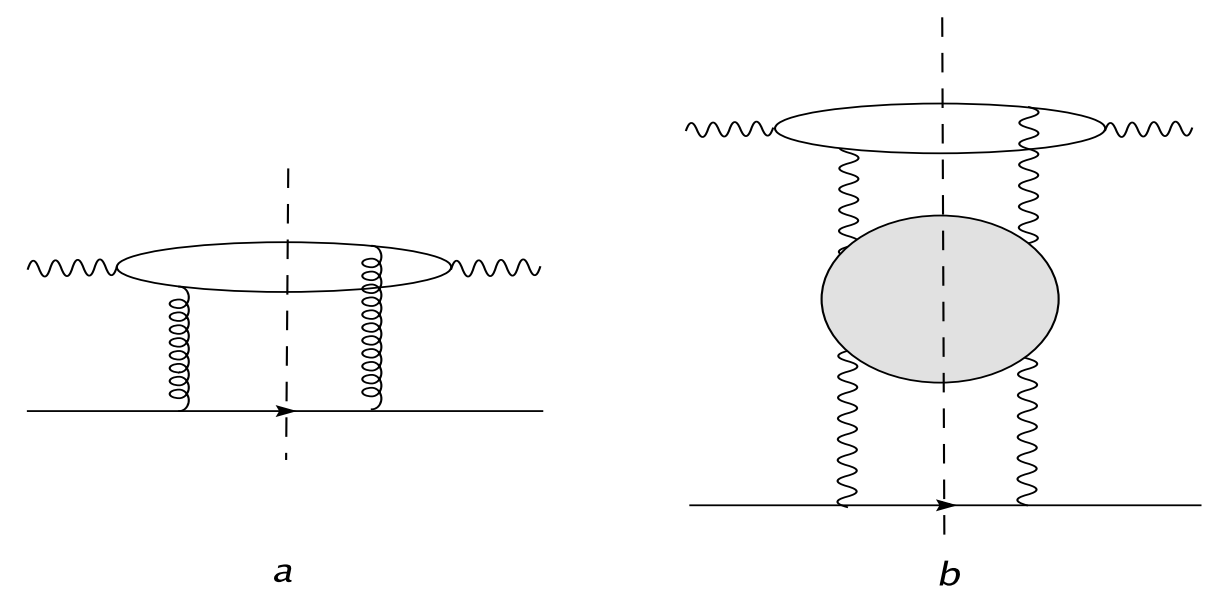

Figure 1: Energy discontinuity of the $2 \rightarrow 2$ process: $\gamma^{*}+q \rightarrow \gamma^{*}+q$.

Finally, a third, $C$-even, piece of the baryonic impact factor appears in which again all three quarks participate. This piece has no counterpart in the quark-antiquark case and, together with the odderon, it makes the baryon really behaving differently from the photon (or the vector meson). The state consists of one reggeized gluon with even signature and two usual odd reggeized gluons. It obeys the BKP evolution in the three Reggeon channel and it decays into four reggeized gluons via a new gauge invariant $3 \rightarrow 4$ reggeized gluon vertex.

The paper is organized as follows. We begin with a short section describing the general framework in which our calculations are carried out. We then (Section 3) turn to the baryon wave function which enters the baryon impact factor. In the following Section 4 we describe the baryon impact factor and its decomposition into the three pieces described above, and in Section 5 we discuss the rapidity evolution of these pieces. Section 6 contains a short discussion of the baryonic impact factor in configuration space, and in Section 7 we analyze the quark-diquark limit of the baryon wave function. Finally, in Section 8 we summarize our results and discuss a few potential implications.

\section{The framework}

In our calculation we will follow the analysis of the scattering of a virtual photon described in $[14,15]$. In leading order the scattering of a virtual photon off a quark is described by the exchange of two gluons. The coupling to the photon is described by the photon impact factor, $D_{2 ; 0}$, which most easily is obtained by the energy discontinuity of a closed quark loop (Fig. 1). Making use of the Regge factorization, the same impact factor can also be used in other elastic scattering processes, e.g. in the scattering of a virtual photon on a heavy onium target. Higher order corrections, in the leading logarithmic approximation, lead to the reggeization of the $t$-channel gluons and to the exchange of a BFKL Pomeron between the photon impact factor and the target.

If one is looking for corrections containing more than two reggeized $t$-channel gluons one has to go beyond the leading logarithmic approximation. In the elastic scattering process $\gamma^{*}+q \rightarrow \gamma^{*}+q$, both leading 


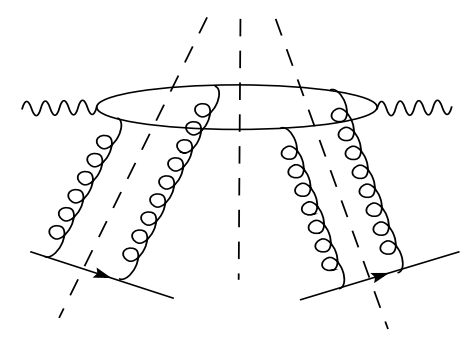

a

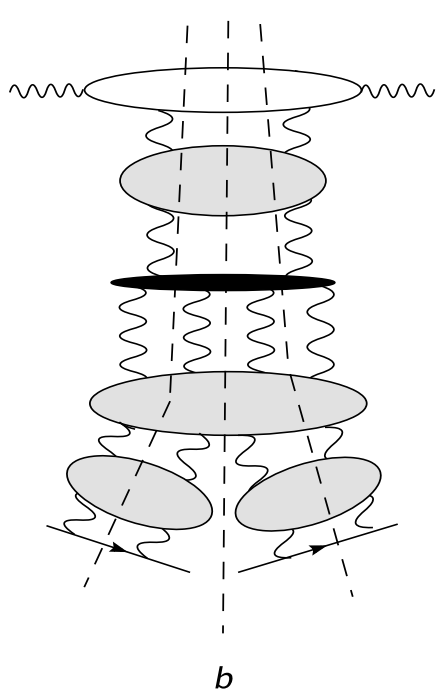

$b$

Figure 2: Multiple energy discontinuities of the $3 \rightarrow 3$ process: $\gamma^{*}+q+q \rightarrow \gamma^{*}+q+q$ : (a) lowest order diagram, (b) two examples of higher order diagrams.

order and NLO corrections retain the structure of a single ladder. A $t$-channel state with four reggeized gluons appears first in NNLO. A convenient way to avoid the complications connected with such a high order calculation is the study of multi-particle processes, e.g the $3 \rightarrow 3$ process $\gamma^{*}+q+q \rightarrow \gamma^{*}+q+q$, the scattering of a virtual photon on two independent quarks (Fig. 2) in the triple Regge limit. This process depends upon three independent energy variables, and the triple energy discontinuity can be easily computed in the approximation where, in each order perturbation theory, the maximal number of large energy logarithms is kept. The lowest order contribution is described by the exchange of four gluons. In higher order, these $t$-channel gluons reggeize and start to interact. As discussed in detail in [14, 15], the all-order result can be cast into the two sets of diagrams shown in Fig. 3.

The first term starts, at the photon impact factor, with a BFKL Green's function, then undergoes the transition into the four gluons and continues with the BKP evolution of the four gluon state. In the large- $N_{c}$ limit, the four gluon state turns into two noninteracting BFKL systems, i.e. we see the beginning of the fan-diagram structure of the BK equation. The second term consists of a simple BFKL Green's function, with higher order splittings of the reggeized gluons at the lower end. As a remarkable feature of this results, in both contributions only two reggeized gluons couple to the photon impact factor, despite the fact that diagrams with four gluons - such as the one shown in Fig. 2a - are included: the apparent 'disappearance' of these contributions is a result of the gluon reggeization which manifests itself in generalized bootstrap relations.

The same strategy can be used to investigate $t$-channel states with higher number of $t$-channel reggeized gluons. For example, six gluons appear in the 8-point amplitude $\gamma^{*}+q+q+q \rightarrow \gamma^{*}+q+q+q$, i.e. the scattering of a virtual photon on three independent quarks. The analysis of this case has been investigated in $[15]$.

Although these results are - initially — derived in the context of a higher order multi-particle processes (e.g. the $3 \rightarrow 3$ scattering process), they nevertheless can be used also in a $2 \rightarrow 2$ process. The diagrams 


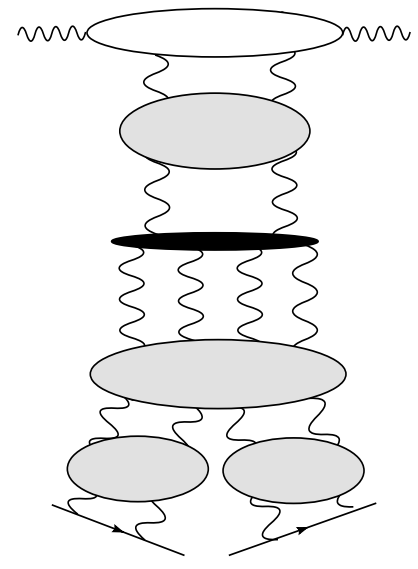

a

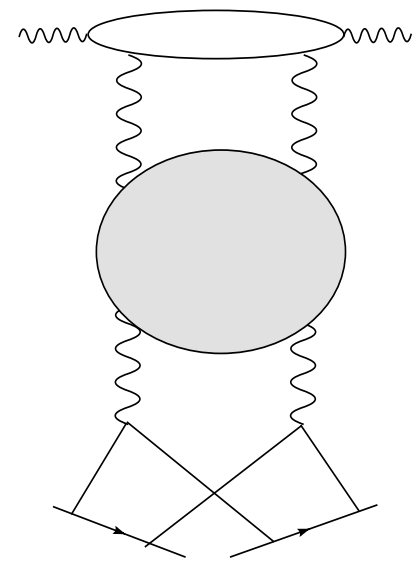

$b$

Figure 3: Decomposition of the sum of all diagrams in Fig. 2b into (a) irreducible and (b) reggeizing pieces.

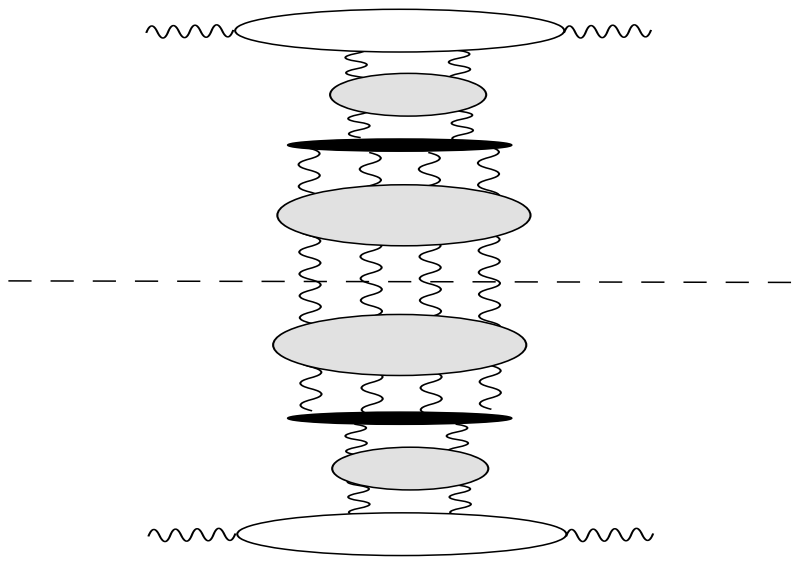

Figure 4: Four gluon contribution to the Reggeon unitarity equation of elastic $\gamma^{*} \gamma^{*}$ scattering.

shown in Fig. 3 satisfy Reggeon unitarity equations in all three $t$-channels. Taking the discontinuity across the four Reggeon state, the partial wave above this can be used to construct the four Reggeon state in the $2 \rightarrow 2$ process shown in Fig. 4 .

In this paper we will apply the same construction, replacing the virtual photon by a three quark system. Modeled by the four fermion operator introduced by Ioffe in the context of the QCD sum rules $[12,19]$, the incoming 'baryon' splits into three quarks which then couple to 2,3 , or 4 gluons. In order to take into account the non-local nature of the incoming baryonic bound state we introduce a form factor: we employ a technique used in the QCD sum rules [19] and use the Borel transform of the perturbative expression [20, 21]. The exponential nature of this form factor also guarantees the convergence of the momentum integrals inside the impact factor. 


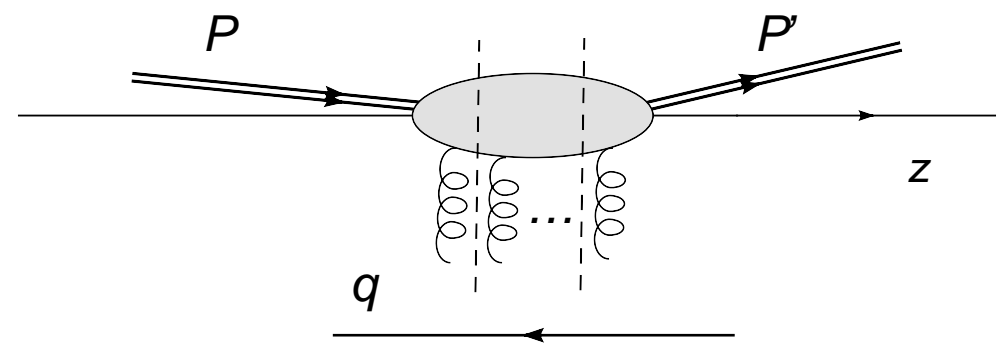

Figure 5: Multiple discontinuity of the impact factor for elastic baryon scattering.

\section{The baryon wave function}

We consider the multiple discontinuity of a non-forward baryon impact factor in elastic high energy scattering. Large momenta are directed along the $z$-axis, and the incoming and outgoing baryons move at small angles with respect to the $z$-axis, as shown in Fig. 5. Their momenta $P, P^{\prime}$ have a large "+" lightcone component, $P^{+}$, and their transverse momenta are denoted by $\boldsymbol{P}, \boldsymbol{P}^{\prime}$, respectively. We introduce the light-like vector $q^{\mu}=(q, 0,0,-q), q^{2}=0$ with $s=(P+q)^{2} \simeq 2 P \cdot q$, and we assume that $s$ is large: $s \gg M^{2}, \boldsymbol{P}^{2}, \boldsymbol{P}^{\prime 2}$. The quark momenta $p_{i}$ are

$$
p_{i}^{\mu}=\left(p_{i}^{0}, \boldsymbol{p}_{i}, p_{i}^{z}\right), \quad p_{i}^{+}=p_{i}^{0}+p_{i}^{z}, \quad p_{i}^{-}=p_{i}^{0}-p_{i}^{z}
$$

For the longitudinal quark momenta it will sometimes be convenient to use the notation

$$
p_{i}^{+}=\alpha_{i} P^{+}, \quad p_{i}^{-}=\beta_{i} q^{-}
$$

We shall use $\hat{p}=\gamma_{\mu} p^{\mu}=\gamma \cdot p$ for contraction of four-vectors and Dirac $\gamma$ matrices. The adopted model of the proton state is defined by

$$
\langle 0|\eta(0)| N(P, \lambda)\rangle=A_{N} w_{\lambda}(P),
$$

where $w_{\lambda}(P)$ is the proton spinor with momentum $P$ and helicity $\lambda$,

$$
\eta(x)=\varepsilon_{\kappa_{1} \kappa_{2} \kappa_{3}}\left[\left(u^{\kappa_{1}}(x)\right)^{T} C \gamma^{\mu} u^{\kappa_{2}}(x)\right] \gamma_{\mu} \gamma_{5} d^{\kappa_{3}}(x)
$$

is the baryonic Ioffe current [12], $C$ is the charge conjugation matrix, and $\kappa_{i}$ are color indices. The Ioffe operator is not the only possible choice of the baryon current, - in the context of distribution amplitudes, the possible baryonic operators for the proton were classified in Ref. [22], and it was shown that the Ioffe current gives a rather good description of baryon form-factors [13]. We therefore chose, as a test case, the Ioffe operator to model the baryonic impact factor. ${ }^{1}$

\footnotetext{
${ }^{1}$ It is worthwhile to stress that our baryon wave functions are different from the distribution amplitudes. In the collinear approach one probes the baryon with a hard external scale, $Q^{2}$, and the baryon structure is represented by series of distribution amplitudes with increasing twist, that is with increasing power-like suppression at large $Q^{2}$. The distribution amplitudes depend on the quark longitudinal momenta, and they obey evolution equations in $\log Q^{2}$. In contrast to that, we are interested in the baryon wave function with full momentum dependence probed at a moderate momentum scale, and the evolution applies to the rapidity of gluons radiated from the baryon impact factor.
} 


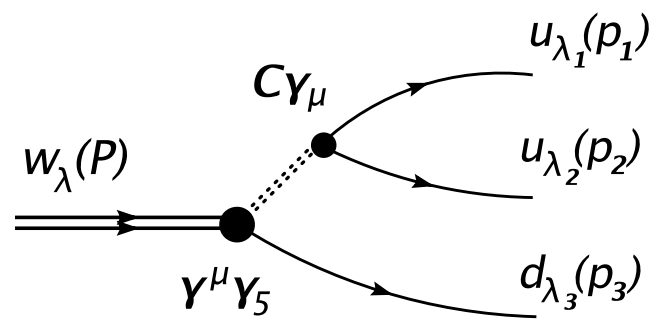

Figure 6: The proton vertex as given by the Ioffe current.

For the calculation of the baryonic impact factor we will need the matrix elements (Fig. 6) in the helicity basis,

$$
\left[\bar{d}_{\lambda_{3}}\left(p_{3}\right) \gamma_{5} \gamma_{\mu} w_{\lambda}(P)\right] \cdot\left[\bar{u}_{\lambda_{1}}\left(p_{1}\right) \gamma^{\mu} C \gamma^{0} u_{\lambda_{2}}^{*}\left(p_{2}\right)\right]
$$

In the second term we can also write:

$$
\left[\bar{u}_{\lambda_{1}}\left(p_{1}\right) \gamma^{\mu} v_{\lambda_{2}}\left(p_{2}\right)\right]
$$

where $v$ (in the Dirac notation) denotes the $v$-spinor of the $u$ quark.

\subsection{The massless quark case}

Using the calculus described by Brodsky and Lepage [23] we compute the Dirac spinor matrix elements. The details of the calculations are described in Appendix A. For simplicity, we start from the massless quark case, and the case of massive quarks will be analyzed afterwards. Thus we obtain:

$$
\begin{gathered}
\frac{\left[\bar{d}_{\lambda}\left(p_{3}\right) \gamma_{5} \gamma_{\mu} w_{\lambda}(P)\right] \cdot\left[\bar{u}_{\lambda_{1}}\left(p_{1}\right) \gamma^{\mu} C \gamma^{0} u_{\lambda_{2}}^{*}\left(p_{2}\right)\right]}{\sqrt{\alpha_{1} \alpha_{2} \alpha_{3}}}= \\
=2 \lambda \delta_{-\lambda_{1}, \lambda_{2}}\left\{\delta_{\lambda_{1}, \lambda}\left[\left(\frac{\boldsymbol{p}_{2}}{\alpha_{2}}-\boldsymbol{P}\right) \cdot\left(\frac{\boldsymbol{p}_{1}}{\alpha_{1}}-\frac{\boldsymbol{p}_{3}}{\alpha_{3}}\right)-i \lambda\left(\frac{\boldsymbol{p}_{2}}{\alpha_{2}}-\boldsymbol{P}\right) \times\left(\frac{\boldsymbol{p}_{1}}{\alpha_{1}}-\frac{\boldsymbol{p}_{3}}{\alpha_{3}}\right)\right]+\right. \\
\left.+\delta_{\lambda_{2}, \lambda}\left[\left(\frac{\boldsymbol{p}_{1}}{\alpha_{1}}-\boldsymbol{P}\right) \cdot\left(\frac{\boldsymbol{p}_{2}}{\alpha_{2}}-\frac{\boldsymbol{p}_{3}}{\alpha_{3}}\right)-i \lambda\left(\frac{\boldsymbol{p}_{1}}{\alpha_{1}}-\boldsymbol{P}\right) \times\left(\frac{\boldsymbol{p}_{2}}{\alpha_{2}}-\frac{\boldsymbol{p}_{3}}{\alpha_{3}}\right)\right]\right\},
\end{gathered}
$$

and,

$$
\begin{gathered}
\frac{\left[\bar{d}_{-\lambda}\left(p_{3}\right) \gamma_{5} \gamma_{\mu} w_{\lambda}(P)\right] \cdot\left[\bar{u}_{\lambda_{1}}\left(p_{1}\right) \gamma^{\mu} C \gamma^{0} u_{\lambda_{2}}^{*}\left(p_{2}\right)\right]}{\sqrt{\alpha_{1} \alpha_{2} \alpha_{3}}}= \\
=2 M \delta_{-\lambda_{1}, \lambda_{2}}\left\{\delta_{\lambda_{1}, \lambda} \boldsymbol{\eta}_{\lambda} \cdot\left(\frac{\boldsymbol{p}_{2}}{\alpha_{2}}-\frac{\boldsymbol{p}_{3}}{\alpha_{3}}\right)+\delta_{\lambda_{2}, \lambda} \boldsymbol{\eta}_{\lambda} \cdot\left(\frac{\boldsymbol{p}_{1}}{\alpha_{1}}-\frac{\boldsymbol{p}_{3}}{\alpha_{3}}\right)\right\},
\end{gathered}
$$

where the transverse complex vector $\boldsymbol{\eta}_{\lambda}$ is defined by

$$
\boldsymbol{\eta}_{\lambda}=(1, i \lambda), \quad \lambda= \pm 1
$$


and $\boldsymbol{P}=\boldsymbol{p}_{1}+\boldsymbol{p}_{2}+\boldsymbol{p}_{3}$ is the transverse momentum of the incoming baryon. The cross product of two transverse vectors $\boldsymbol{p}_{1}=\left(p_{1}^{x}, p_{1}^{y}\right)$ and $\boldsymbol{p}_{2}=\left(p_{2}^{x}, p_{2}^{y}\right)$ should be understood as a number $\boldsymbol{p}_{1} \times \boldsymbol{p}_{2}=p_{1}^{x} p_{2}^{y}-p_{1}^{y} p_{2}^{x}$. It turns out that formula (7) may be re-expressed in a more compact form, by using the vectors $\boldsymbol{\eta}_{\lambda}$ with the following identity:

$$
\left(\boldsymbol{p}_{1} \cdot \boldsymbol{\eta}_{\lambda}\right)\left(\boldsymbol{p}_{2} \cdot \boldsymbol{\eta}_{-\lambda}\right)=\left(p_{1}^{x}+i \lambda p_{1}^{y}\right)\left(p_{2}^{x}-i \lambda p_{2}^{y}\right)=p_{1}^{x} p_{2}^{x}+p_{1}^{y} p_{2}^{y}+i \lambda\left(p_{1}^{y} p_{2}^{x}-p_{1}^{x} p_{2}^{y}\right)=\boldsymbol{p}_{1} \cdot \boldsymbol{p}_{2}-i \lambda \boldsymbol{p}_{1} \times \boldsymbol{p}_{2},
$$

which holds for any pair of transverse vectors, $\boldsymbol{p}_{1}$ and $\boldsymbol{p}_{2}$. Using this relation one gets:

$$
\begin{gathered}
\frac{\left[\bar{d}_{\lambda}\left(p_{3}\right) \gamma_{5} \gamma_{\mu} w_{\lambda}(P)\right] \cdot\left[\bar{u}_{\lambda_{1}}\left(p_{1}\right) \gamma^{\mu} C \gamma^{0} u_{\lambda_{2}}^{*}\left(p_{2}\right)\right]}{\sqrt{\alpha_{1} \alpha_{2} \alpha_{3}}}= \\
=2 \delta_{-\lambda_{1}, \lambda_{2}} \times\left\{\delta_{\lambda_{1}, \lambda}\left[\boldsymbol{\eta}_{\lambda} \cdot\left(\frac{\boldsymbol{p}_{2}}{\alpha_{2}}-\boldsymbol{P}\right)\right]\left[\boldsymbol{\eta}_{-\lambda} \cdot\left(\frac{\boldsymbol{p}_{1}}{\alpha_{1}}-\frac{\boldsymbol{p}_{3}}{\alpha_{3}}\right)\right]+\right. \\
\left.\delta_{\lambda_{2}, \lambda}\left[\boldsymbol{\eta}_{\lambda} \cdot\left(\frac{\boldsymbol{p}_{1}}{\alpha_{1}}-\boldsymbol{P}\right)\right]\left[\boldsymbol{\eta}_{-\lambda} \cdot\left(\frac{\boldsymbol{p}_{2}}{\alpha_{2}}-\frac{\boldsymbol{p}_{3}}{\alpha_{3}}\right)\right]\right\} .
\end{gathered}
$$

In what follows, we shall express all formulae in this compact notation.

Next we couple a gluon of momentum $k=\beta q+\boldsymbol{k}$ to one of the quark lines with momentum $p_{i}$ (Fig. 7). Fixing the momenta of the outgoing quarks at $p_{1}, p_{2}$, and $p_{3}$, the quark line to the left of the gluon vertex carries momentum $p_{i}-k$. Using, at the gluon vertex, the eikonal approximation, one arrives at the spinorial factor $\hat{q}$. With $2 p_{i} \cdot q=\alpha_{i} s \gg \boldsymbol{p}_{i}^{2}, \boldsymbol{k}^{2}$, etc. , one obtains, for the upper $u$ quark,

$$
\bar{u}\left(p_{1}\right) \hat{q}\left(\hat{p_{1}}-\hat{k}\right)=2 p_{1} \cdot q \bar{u}\left(p_{1}-k\right)+\ldots,
$$

where ... stands for terms which are power suppressed in $s$. An analogous expression holds for the $d$ quark, whereas for the second $u$ quark we use:

$$
\left(\hat{p_{2}}-\hat{k}\right) \hat{q} u^{*}\left(p_{2}\right)=2 p_{2} \cdot q u^{*}\left(p_{2}-k\right)+\ldots
$$

As a result, on the r.h.s. of Eqs. (12) and (13), the transverse momentum of the quark spinor coincides with the transverse momentum of the internal quark line next to the baryon vertex. The sum of the outgoing transverse momenta equals

$$
\boldsymbol{p}_{1}+\boldsymbol{p}_{2}+\boldsymbol{p}_{3}=\boldsymbol{P}+\boldsymbol{k}
$$

Matrix elements corresponding to multi-gluon couplings to spinor lines may be simplified by iterating Eq. (12) in the following way:

$$
\bar{u}(p) \hat{q}\left[\gamma \cdot\left(p-k_{1}\right)\right] \hat{q} \ldots \hat{q}\left[\gamma \cdot\left(p-k_{1}-\ldots-k_{n}\right)\right] \simeq(2 p \cdot q)^{n} \bar{u}\left(p-k_{1}-\ldots-k_{n}\right) .
$$

For completeness, we remind that, in the case of an outgoing antiquark, an additional minus sign appears:

$$
-(\hat{p}-\hat{k}) \hat{q} v(p)=-2 p \cdot q v(p-k)+\ldots
$$

This minus sign is due to the opposite direction of the momentum along the antifermion line. Similarly:

$$
\left[-\gamma \cdot\left(p-k_{1}-\ldots-k_{n}\right)\right] \hat{q} \ldots \hat{q}\left[-\gamma \cdot\left(p-k_{1}\right)\right] \hat{q} v(p) \simeq(-2 p \cdot q)^{n} v\left(p-k_{1}-\cdots-k_{n}\right)
$$




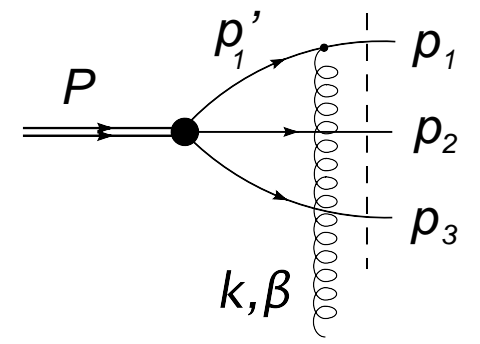

a)

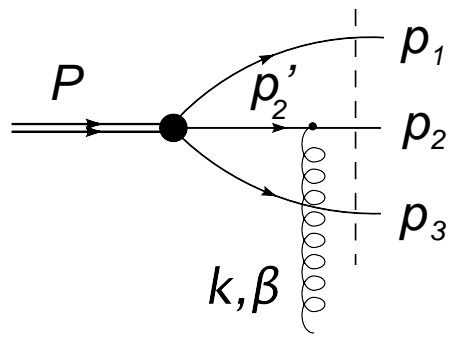

b)

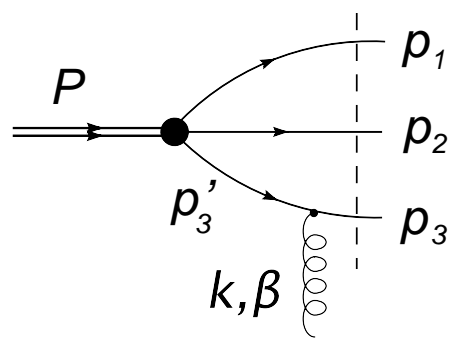

c)

Figure 7: Single gluon coupling to the baryon $\rightarrow X$ transition.

This change in sign plays a crucial rôle in the photon impact factor [7].

We are now ready to describe the amplitude for the process: baryon + gluon $\rightarrow 3$ quarks, defined by the diagrams shown in Fig. 7. We define the shifted momentum of the upper quark

$$
\boldsymbol{p}_{1}^{\prime}=\boldsymbol{p}_{1}-\boldsymbol{k}
$$

with

$$
\boldsymbol{p}_{1}^{\prime}+\boldsymbol{p}_{2}+\boldsymbol{p}_{3}=\boldsymbol{P}
$$

and use

$$
{p_{1}^{\prime}}^{2}=\alpha_{1}\left(M^{2}+\boldsymbol{P}^{2}-\frac{\boldsymbol{p}_{1}^{\prime 2}}{\alpha_{1}}-\frac{\boldsymbol{p}_{2}^{2}}{\alpha_{2}}-\frac{\boldsymbol{p}_{3}{ }^{2}}{\alpha_{3}}\right),
$$

(and analogous expressions for the gluon coupling to quark lines 2 and 3). We introduce the amplitudes

$$
\begin{aligned}
\Theta_{\lambda}^{\left(\lambda_{1}, \lambda_{2}\right) \lambda}\left(\left\{\alpha_{i}\right\},\left\{\boldsymbol{p}_{1}, \boldsymbol{p}_{2}, \boldsymbol{p}_{3}\right\}, \boldsymbol{P}\right)= & \lambda \mathcal{N}_{\Theta} \frac{2 \sqrt{\alpha_{1} \alpha_{2} \alpha_{3}}}{M^{2}+\boldsymbol{P}^{2}-\frac{\boldsymbol{p}_{1}^{2}}{\alpha_{1}}-\frac{\boldsymbol{p}_{2}^{2}}{\alpha_{2}}-\frac{\boldsymbol{p}_{3}^{2}}{\alpha_{3}}} \delta_{-\lambda_{1}, \lambda_{2}} \delta^{(2)}\left(\boldsymbol{p}_{1}+\boldsymbol{p}_{2}+\boldsymbol{p}_{3}-\boldsymbol{P}\right) \times \\
\times & \left\{\delta_{\lambda_{1}, \lambda}\left[\boldsymbol{\eta}_{\lambda} \cdot\left(\frac{\boldsymbol{p}_{2}}{\alpha_{2}}-\boldsymbol{P}\right)\right]\left[\boldsymbol{\eta}_{-\lambda} \cdot\left(\frac{\boldsymbol{p}_{1}}{\alpha_{1}}-\frac{\boldsymbol{p}_{3}}{\alpha_{3}}\right)\right]+\right. \\
& \left.\delta_{\lambda_{2}, \lambda}\left[\boldsymbol{\eta}_{\lambda} \cdot\left(\frac{\boldsymbol{p}_{1}}{\alpha_{1}}-\boldsymbol{P}\right)\right]\left[\boldsymbol{\eta}_{-\lambda} \cdot\left(\frac{\boldsymbol{p}_{2}}{\alpha_{2}}-\frac{\boldsymbol{p}_{3}}{\alpha_{3}}\right)\right]\right\} \\
\Theta_{\lambda}^{\left(\lambda_{1}, \lambda_{2}\right)-\lambda}\left(\left\{\alpha_{i}\right\},\left\{\boldsymbol{p}_{1}, \boldsymbol{p}_{2}, \boldsymbol{p}_{3}\right\} ; \boldsymbol{P}\right)= & \mathcal{N}_{\Theta} \frac{2 M \sqrt{\alpha_{1} \alpha_{2} \alpha_{3}}}{M^{2}+\boldsymbol{P}^{2}-\frac{\boldsymbol{p}_{1}^{2}}{\alpha_{1}}-\frac{\boldsymbol{p}_{2}^{2}}{\alpha_{2}}-\frac{\boldsymbol{p}_{3}^{2}}{\alpha_{3}}} \delta_{-\lambda_{1}, \lambda_{2}} \delta^{(2)}\left(\boldsymbol{p}_{1}+\boldsymbol{p}_{2}+\boldsymbol{p}_{3}-\boldsymbol{P}\right) \times \\
\times & \left\{\delta_{\lambda_{1}, \lambda} \boldsymbol{\eta}_{\lambda} \cdot\left(\frac{\boldsymbol{p}_{2}}{\alpha_{2}}-\frac{\boldsymbol{p}_{3}}{\alpha_{3}}\right)+\delta_{\lambda_{2}, \lambda} \boldsymbol{\eta}_{\lambda} \cdot\left(\frac{\boldsymbol{p}_{1}}{\alpha_{1}}-\frac{\boldsymbol{p}_{3}}{\alpha_{3}}\right)\right\} .
\end{aligned}
$$

Here the upper three indices of $\Theta$ denote the helicities of the upper two u quarks with momenta $\boldsymbol{p}_{1}$ and $\boldsymbol{p}_{2}$, and the lower $\mathrm{d}$ quark with momentum $\boldsymbol{p}_{3}$, respectively. The subscript refers to the helicity $\lambda$ of the incoming baryon. We leave the normalization constant $\mathcal{N}_{\Theta}$ unspecified here; the normalization will be fixed at the level of baryon wave function. The amplitudes for the diagrams shown in Figs. 7a-7c are then simply obtained from (21), (22) by the replacements $\boldsymbol{p}_{1} \rightarrow \boldsymbol{p}_{1}^{\prime}, \boldsymbol{p}_{2}, \rightarrow \boldsymbol{p}_{2}^{\prime}$ and $\boldsymbol{p}_{3} \rightarrow \boldsymbol{p}_{3}^{\prime}$, respectively. $^{2}$

\footnotetext{
${ }^{2}$ To be precise, the functions $\Theta$ give the momentum dependent part of the scattering amplitudes, up to a global normalization factor, that is proportional to the strong coupling constant $g$. Obviously, the color factors are not accounted for in (21) and (22), — they will be treated explicitly later on.
} 
Note that, for each of the three diagrams, the denominator is just the energy denominator in noncovariant perturbation theory, for instance one obtains for Fig. 7a,

$$
E_{\text {baryon }}-E_{3 \text { quark }}=\frac{1}{P^{+}}\left(M^{2}+P^{2}-\frac{p_{1}^{\prime 2}}{\alpha_{1}}-\frac{\boldsymbol{p}_{2}^{2}}{\alpha_{2}}-\frac{\boldsymbol{p}_{3}^{2}}{\alpha_{3}}\right) .
$$

The amplitudes should be invariant under Lorentz boosts in the transverse directions, parametrized by a four-velocity $u^{\mu} \simeq(1, \boldsymbol{u}, 0),|\boldsymbol{u}| \ll 1$ :

$$
\boldsymbol{p}_{i} \rightarrow \boldsymbol{p}_{i}^{\prime} \simeq \boldsymbol{p}_{i}+\frac{1}{2} p_{i}^{+} \boldsymbol{u}, \quad p_{i}^{+} \rightarrow p_{i}^{+} \simeq p_{i}^{+}
$$

The numerators are manifestly invariant under these transformations, and the denominators may be also rewritten in an explicitly invariant form using the identity

$$
-\boldsymbol{P}^{2}+\frac{\boldsymbol{p}_{1}^{2}}{\alpha_{1}}+\frac{\boldsymbol{p}_{2}^{2}}{\alpha_{2}}+\frac{\boldsymbol{p}_{3}^{2}}{\alpha_{3}}=\alpha_{1} \alpha_{2}\left(\frac{\boldsymbol{p}_{1}}{\alpha_{1}}-\frac{\boldsymbol{p}_{2}}{\alpha_{2}}\right)^{2}+\alpha_{1} \alpha_{3}\left(\frac{\boldsymbol{p}_{3}}{\alpha_{3}}-\frac{\boldsymbol{p}_{1}}{\alpha_{1}}\right)^{2}+\alpha_{2} \alpha_{3}\left(\frac{\boldsymbol{p}_{2}}{\alpha_{2}}-\frac{\boldsymbol{p}_{3}}{\alpha_{3}}\right)^{2},
$$

or

$$
-\boldsymbol{P}^{2}+\frac{\boldsymbol{p}_{1}^{2}}{\alpha_{1}}+\frac{\boldsymbol{p}_{2}^{2}}{\alpha_{2}}+\frac{\boldsymbol{p}_{3}^{2}}{\alpha_{3}}=\frac{\left(\boldsymbol{p}_{1}-\alpha_{1} \boldsymbol{P}\right)^{2}}{\alpha_{1}}+\frac{\left(\boldsymbol{p}_{2}-\alpha_{2} \boldsymbol{P}\right)^{2}}{\alpha_{2}}+\frac{\left(\boldsymbol{p}_{3}-\alpha_{3} \boldsymbol{P}\right)^{2}}{\alpha_{3}} .
$$

The denominators have poles for the invariant mass of the three-quark system equal to the proton transverse mass. Clearly, this is a consequence of using a point-like vertex for the proton-quark coupling and neglecting the bound state effects. These effects cannot be described within perturbative QCD and should be modeled. Following $[19,20]$ we propose a model that preserves Lorentz and helicity structures of the perturbative expressions, where the bound state effects are absorbed into the Borel transform.

The Borel transform of a function $f(s)$ is defined in the standard way:

$$
\mathcal{B}_{s}[f]\left(M_{B}^{2}\right)=\lim _{n \rightarrow \infty} \frac{s^{n+1}}{n !}\left(-\frac{d}{d s}\right)^{n} f(s), \quad s \rightarrow \infty, \quad s / n \rightarrow M_{B}^{2},
$$

where $M_{B}$ is the Borel parameter. In order to model the baryon scattering amplitude we shall apply two independent Borel transforms w.r.t. the negative virtualities: $Q^{2}=-P^{2}$ of the incoming and $Q^{\prime 2}=-P^{\prime 2}$ of the outgoing baryon, to the perturbative amplitudes obtained with the point-like vertex. Formulae (21) and (22) were presented for $P^{2}=M^{2}$. The corresponding formulae for general virtualities are obtained by substitutions $M^{2} \rightarrow P^{2}$ in the denominators. In the baryon impact factor, the virtuality $P^{2}$ appears only in the energy denominator of the vertex amplitude $\Theta_{\lambda}^{\left(\lambda_{1}, \lambda_{2}\right) \lambda_{3}}\left(\left\{\alpha_{i}\right\},\left\{\boldsymbol{p}_{i}\right\} ; \boldsymbol{P}\right)$ of the incoming baryon, and the virtuality $P^{\prime 2}$ only in the denominator of the amplitude $\left[\Theta_{\lambda}^{\left(\lambda_{1}, \lambda_{2}\right) \lambda_{3}}\left(\left\{\alpha_{i}\right\},\left\{\boldsymbol{p}_{i}\right\} ; \boldsymbol{P}^{\prime}\right)\right]^{*}$ of the outgoing state (see Sec. 4 for more details). Therefore the two Borel transforms may be performed independently for each $\Theta$, that is already at the level of the baryon wave function. Thus we evaluate

$$
\mathcal{B}_{Q^{2}}\left[\frac{1}{Q^{2}+M_{X}^{2}}\right]\left(M_{B}^{2}\right)=\exp \left(-M_{X}^{2} / M_{B}^{2}\right)
$$

This result, applied to the amplitudes $\Theta$, leads to the substitution

$$
\frac{1}{P^{2}+P^{2}-\sum_{i=1}^{3} \frac{p_{i}^{2}}{\alpha_{i}}} \longrightarrow-\exp \left[-\frac{1}{M_{B}^{2}}\left(\sum_{i=1}^{3} \frac{\boldsymbol{p}_{i}^{2}}{\alpha_{i}}-\boldsymbol{P}^{2}\right)\right]
$$


Before we complete the model we shall perform some simplifications. We shall absorb into the wave functions a phase space factor $\left(\alpha_{1} \alpha_{2} \alpha_{3}\right)^{-1}$ that appears in the baryon impact factor as a result of on-massshell conditions of the cut quark lines. In this way, the factor $\sqrt{\alpha_{1} \alpha_{2} \alpha_{3}}$ present in the amplitudes $\Theta$ will be removed from the wave functions. Obviously, the integration measure will be suitably modified as well. For simplicity, we introduce a normalization constant, $\mathcal{N}$, of the wave function that will be fixed later. Thus, we choose the natural value of the Borel parameter $M_{B}=M$ and obtain a model of the baryon wave function,

$$
\begin{aligned}
\Psi_{\lambda}^{\left(\lambda_{1}, \lambda_{2}\right) \lambda}\left(\left\{\alpha_{i}\right\},\left\{\boldsymbol{p}_{i}\right\} ; \boldsymbol{P}\right) & =\lambda \mathcal{N} e^{-\frac{1}{M^{2}}\left(-\boldsymbol{P}^{2}+\frac{\boldsymbol{p}_{1}{ }^{2}}{\alpha_{1}}+\frac{\boldsymbol{p}_{2}{ }^{2}}{\alpha_{2}}+\frac{\boldsymbol{p}_{3}{ }^{2}}{\alpha_{3}}\right)} \delta_{-\lambda_{1}, \lambda_{2}} \delta^{(2)}\left(\boldsymbol{p}_{1}+\boldsymbol{p}_{2}+\boldsymbol{p}_{3}-\boldsymbol{P}\right) \times \\
& \times\left\{\delta_{\lambda_{1}, \lambda}\left[\boldsymbol{\eta}_{\lambda} \cdot\left(\frac{\boldsymbol{p}_{2}}{\alpha_{2}}-\boldsymbol{P}\right)\right]\left[\boldsymbol{\eta}_{-\lambda} \cdot\left(\frac{\boldsymbol{p}_{1}}{\alpha_{1}}-\frac{\boldsymbol{p}_{3}}{\alpha_{3}}\right)\right]+\right. \\
& \left.+\delta_{\lambda_{2}, \lambda}\left[\boldsymbol{\eta}_{\lambda} \cdot\left(\frac{\boldsymbol{p}_{1}}{\alpha_{1}}-\boldsymbol{P}\right)\right]\left[\boldsymbol{\eta}_{-\lambda} \cdot\left(\frac{\boldsymbol{p}_{2}}{\alpha_{2}}-\frac{\boldsymbol{p}_{3}}{\alpha_{3}}\right)\right]\right\}, \\
\Psi_{\lambda}^{\left(\lambda_{1}, \lambda_{2}\right)-\lambda}\left(\left\{\alpha_{i}\right\},\left\{\boldsymbol{p}_{i}\right\} ; \boldsymbol{P}\right) & =\mathcal{N} e^{-\frac{1}{M^{2}}\left(-\boldsymbol{P}^{2}+\frac{\boldsymbol{p}_{1}{ }^{2}}{\alpha_{1}}+\frac{\boldsymbol{p}_{2}}{\alpha_{2}}+\frac{\boldsymbol{p}_{3}{ }^{2}}{\alpha_{3}}\right)} \delta_{-\lambda_{1}, \lambda_{2}} \delta^{(2)}\left(\boldsymbol{p}_{1}+\boldsymbol{p}_{2}+\boldsymbol{p}_{3}-\boldsymbol{P}\right) \times \\
& \times \quad M\left\{\delta_{\lambda_{1}, \lambda} \boldsymbol{\eta}_{\lambda} \cdot\left(\frac{\boldsymbol{p}_{2}}{\alpha_{2}}-\frac{\boldsymbol{p}_{3}}{\alpha_{3}}\right)+\delta_{\lambda_{2}, \lambda} \boldsymbol{\eta}_{\lambda} \cdot\left(\frac{\boldsymbol{p}_{1}}{\alpha_{1}}-\frac{\boldsymbol{p}_{3}}{\alpha_{3}}\right)\right\} .
\end{aligned}
$$

Clearly, the functions $\Psi$ given by Eqs. (30) and (31) are symmetric under the interchange of the $u$ quarks, labeled by 1 and 2 . When combined with the anti-symmetry in the color degrees of freedom it implies that the full wave function is anti-symmetric under interchange of the $u$ quarks, as it must be. Interestingly enough, a similar Gaussian form of the wave function was proposed long ago [24] and it was shown to provide a good description of the nucleon form-factor data $[25,26]$. An important difference of our model, however, is the presence of angular momenta of the quarks. The baryon angular momentum structure following from the model is most transparent in the coordinate representation and will be discussed is Section 6 .

The above derivation of the baryon wave function is based on perturbative QCD methods combined with the Borel transform technique. Clearly, we are not able to control the accuracy of this procedure for the proton as it is a genuine non-perturbative object. Therefore the obtained wave functions can be only considered as a theoretically inspired model of the proton wave function. Therefore, in the next part we give the formulae for the wave function of a baryon consisting of three quarks with the same mass $m$, coming in two different flavors. These formulae will permit to consider the fictitious case of a large quark mass, for which the baryon becomes heavy and small, and the perturbative computation of its wave function and scattering is formally justified.

\subsection{Massive quarks}

We now apply the procedure described in the previous section to the case of the massive quarks. We skip the details of the derivation and present the result for the helicity amplitudes $\Theta$ of the transition: baryon to quarks, in which all three quarks were assumed to have the mass $m$ : 


$$
\begin{aligned}
& \Theta_{\lambda}^{\left(\lambda_{1}, \lambda_{2}\right) \lambda_{3}}\left(\left\{\alpha_{i}\right\},\left\{\boldsymbol{p}_{i}\right\}, \boldsymbol{P}\right)=\mathcal{N}_{\Theta} \frac{2 \sqrt{\alpha_{1} \alpha_{2} \alpha_{3}}}{M^{2}+\boldsymbol{P}^{2}-\frac{\boldsymbol{p}_{1}^{2}+m^{2}}{\alpha_{1}}-\frac{\boldsymbol{p}_{2}^{2}+m^{2}}{\alpha_{2}}-\frac{\boldsymbol{p}_{3}^{2}+m^{2}}{\alpha_{3}}} \delta^{(2)}\left(\boldsymbol{p}_{1}+\boldsymbol{p}_{2}+\boldsymbol{p}_{3}-\boldsymbol{P}\right) \times \\
& \quad \times \quad\left\{\delta_{\lambda, \lambda_{1}} \delta_{\lambda, \lambda_{2}} \delta_{\lambda, \lambda_{3}} m \frac{\alpha_{1}+\alpha_{2}}{\alpha_{1} \alpha_{2}}\left(\frac{\boldsymbol{p}_{3}}{\alpha_{3}}-\frac{\boldsymbol{p}_{1}+\boldsymbol{p}_{2}}{\alpha_{1}+\alpha_{2}}\right) \cdot \boldsymbol{\eta}_{-\lambda}+\right. \\
& +\lambda \delta_{\lambda, \lambda_{1}} \delta_{\lambda, \lambda_{2}} \delta_{\lambda,-\lambda_{3}} m \frac{\alpha_{1}+\alpha_{2}}{\alpha_{1} \alpha_{2}}\left(M-\frac{m}{\alpha_{3}}\right)+ \\
& +\delta_{\lambda,-\lambda_{1}} \delta_{\lambda,-\lambda_{2}} \delta_{\lambda, \lambda_{3}} m \frac{\alpha_{1}+\alpha_{2}}{\alpha_{1} \alpha_{2}}\left(\frac{\boldsymbol{p}_{1}+\boldsymbol{p}_{2}}{\alpha_{1}+\alpha_{2}}-\boldsymbol{P}\right) \cdot \boldsymbol{\eta}_{\lambda}+ \\
& +\lambda \delta_{\lambda, \lambda_{1}} \delta_{\lambda,-\lambda_{2}} \delta_{\lambda, \lambda_{3}}\left[\left(\frac{\boldsymbol{p}_{2}}{\alpha_{2}}-\boldsymbol{P}\right) \cdot \boldsymbol{\eta}_{\lambda}\left(\frac{\boldsymbol{p}_{1}}{\alpha_{1}}-\frac{\boldsymbol{p}_{3}}{\alpha_{3}}\right) \cdot \boldsymbol{\eta}_{-\lambda}+m\left(\frac{M}{\alpha_{3}}-\frac{m}{\alpha_{1} \alpha_{2}}\right)\right]+ \\
& +\lambda \delta_{\lambda,-\lambda_{1}} \delta_{\lambda, \lambda_{2}} \delta_{\lambda, \lambda_{3}}\left[\left(\frac{\boldsymbol{p}_{1}}{\alpha_{1}}-\boldsymbol{P}\right) \cdot \boldsymbol{\eta}_{\lambda}\left(\frac{\boldsymbol{p}_{2}}{\alpha_{2}}-\frac{\boldsymbol{p}_{3}}{\alpha_{3}}\right) \cdot \boldsymbol{\eta}_{-\lambda}+m\left(\frac{M}{\alpha_{3}}-\frac{m}{\alpha_{1} \alpha_{2}}\right)\right]+ \\
& +\delta_{\lambda, \lambda_{1}} \delta_{\lambda,-\lambda_{2}} \delta_{\lambda,-\lambda_{3}}\left[M\left(\frac{\boldsymbol{p}_{2}}{\alpha_{2}}-\frac{\boldsymbol{p}_{3}}{\alpha_{3}}\right) \cdot \boldsymbol{\eta}_{\lambda}+m \frac{1-\alpha_{3}}{\alpha_{3}}\left(\frac{\boldsymbol{p}_{2}}{\alpha_{2}}-\frac{\boldsymbol{p}_{1}+\boldsymbol{p}_{2}}{\alpha_{1}+\alpha_{2}}\right) \cdot \boldsymbol{\eta}_{\lambda}\right]+ \\
& \left.+\delta_{\lambda,-\lambda_{1}} \delta_{\lambda, \lambda_{2}} \delta_{\lambda,-\lambda_{3}}\left[M\left(\frac{\boldsymbol{p}_{1}}{\alpha_{1}}-\frac{\boldsymbol{p}_{3}}{\alpha_{3}}\right) \cdot \boldsymbol{\eta}_{\lambda}+m \frac{1-\alpha_{3}}{\alpha_{3}}\left(\frac{\boldsymbol{p}_{1}}{\alpha_{1}}-\frac{\boldsymbol{p}_{1}+\boldsymbol{p}_{2}}{\alpha_{1}+\alpha_{2}}\right) \cdot \boldsymbol{\eta}_{\lambda}\right]\right\} .
\end{aligned}
$$

Note, that using relation (10) and taking $m \rightarrow 0$ one easily recovers formulae (21) and (22). The above formulae are promoted to the baryon wave functions $\Psi$, by going through the same steps as in the massless case, i.e. using the Borel transform and absorbing the $\alpha$ factors into the phase space factor. The final expressions for the wave functions are obtained from Eq. (32) by the replacement which combines both steps:

$$
\mathcal{N}_{\Theta} \frac{2 \sqrt{\alpha_{1} \alpha_{2} \alpha_{3}}}{M^{2}+\boldsymbol{P}^{2}-\sum_{i=1}^{3} \frac{\boldsymbol{p}_{i}^{2}+m^{2}}{\alpha_{i}}} \longrightarrow \mathcal{N} \exp \left[-\frac{1}{M^{2}}\left(\sum_{i=1}^{3} \frac{\boldsymbol{p}_{i}^{2}+m^{2}}{\alpha_{i}}-\boldsymbol{P}^{2}\right)\right]
$$

\section{Baryon impact factors}

\subsection{General structure}

The amplitudes $\Psi$ may be combined with their complex conjugates to obtain the baryon impact factor. For the case of two gluons coupled to lines 3 and 1 we illustrate one example in Fig. 8. It was shown in the previous section that, in the high energy limit the spinorial part of the multiple discontinuity can be expressed in terms of universal matrix elements given by Eqs. (7) and (8), where the momenta of the quarks are evaluated at the quark-proton vertex. Also the denominator is determined by the virtuality of the quark to which the first gluon couples, and the virtuality can expressed in terms of the momenta of the quarks at the proton vertex. Thus, the impact factor can be obtained from overlap integrals, i.e. products of wave 


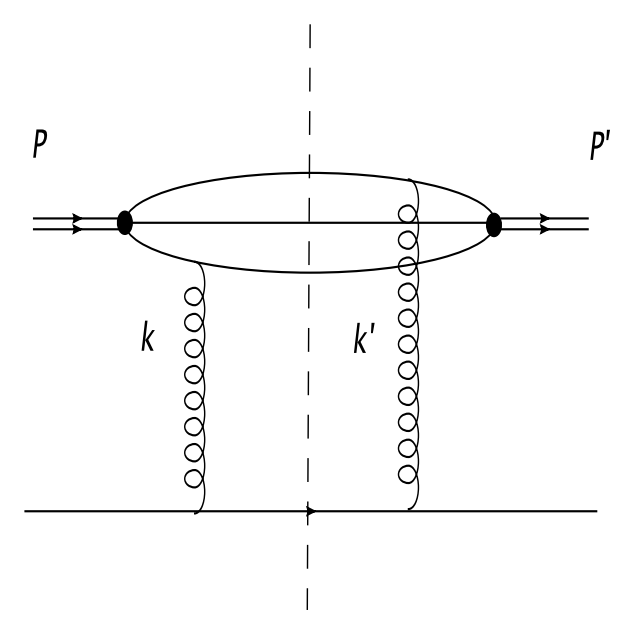

Figure 8: A contribution to the two gluon exchange in baryon-quark scattering.

functions with suitably adjusted momenta. As an example, we specify the overlap function corresponding Fig. 8:

$$
\begin{aligned}
\mathcal{F}^{\lambda \lambda^{\prime}}\left(\left\{\boldsymbol{k}, \boldsymbol{k}^{\prime}\right\} ; \boldsymbol{P}, \boldsymbol{P}^{\prime}\right)=\sum_{\lambda_{1}, \lambda_{2}, \lambda_{3}} \int\left[d^{2} \boldsymbol{p}_{i}\right]\left[d \alpha_{i}\right] & {\left[\Psi_{\lambda^{\prime}}^{\left(\lambda_{1}, \lambda_{2}\right) \lambda_{3}}\left(\left\{\alpha_{i}\right\},\left\{\boldsymbol{p}_{1}^{\prime}, \boldsymbol{p}_{2}, \boldsymbol{p}_{3}\right\} ; \boldsymbol{P}^{\prime}\right)\right]^{*} } \\
& \times \Psi_{\lambda}^{\left(\lambda_{1}, \lambda_{2}\right) \lambda_{3}}\left(\left\{\alpha_{i}\right\},\left\{\boldsymbol{p}_{1}, \boldsymbol{p}_{2}, \boldsymbol{p}_{3}^{\prime}\right\} ; \boldsymbol{P}\right),
\end{aligned}
$$

with the integration measure

$$
\left[d^{2} \boldsymbol{p}_{i}\right]=d^{2} \boldsymbol{p}_{1} d^{2} \boldsymbol{p}_{2} d^{2} \boldsymbol{p}_{3}, \quad\left[d \alpha_{i}\right]=d \alpha_{1} d \alpha_{2} d \alpha_{3} \delta\left(\alpha_{1}+\alpha_{2}+\alpha_{3}-1\right) .
$$

In the overlap functions $\mathcal{F}^{\lambda \lambda^{\prime}}$, the upper helicity labels refer to the incoming and outgoing baryon states, respectively. Analogous overlap functions are defined for the other gluon couplings, and for the full impact factor we will have to sum over all diagrams. When evaluating the sum over the intermediate helicities $\lambda_{1}, \lambda_{2}$, and $\lambda_{3}$ and summing over all diagrams, one finds, for the forward direction $\boldsymbol{P}=\boldsymbol{P}^{\prime}=0$, helicity conservation, i.e. the impact factor vanishes for $\lambda=-\lambda^{\prime}$.

Before including the remaining energy integrals and the color factors we generalize to the case of 3 and 4 $t$-channel gluons. As outlined in Section 2, we have to consider multiple energy discontinuities. An example is shown in Fig. 9. For one of the discontinuity lines (in the case of Fig. 9, the central line) we fix the intermediate quark momenta and denote them by $\boldsymbol{p}_{1}, \boldsymbol{p}_{2}, \boldsymbol{p}_{3}$. The corresponding overlap function (Fig. 10) takes the form

$$
\begin{aligned}
\mathcal{F}^{\lambda \lambda^{\prime}}\left(\left\{\boldsymbol{k}_{i}, \boldsymbol{k}_{j}^{\prime}\right\} ; \boldsymbol{P}, \boldsymbol{P}^{\prime}\right)=\sum_{\lambda_{1}, \lambda_{2}, \lambda_{3}} \int\left[d^{2} \boldsymbol{p}_{i}\right]\left[d \alpha_{i}\right] & {\left[\Psi_{\lambda^{\prime}}^{\left(\lambda_{1}, \lambda_{2}\right) \lambda_{3}}\left(\left\{\alpha_{i}\right\},\left\{\boldsymbol{p}_{1}+\boldsymbol{k}_{1}^{\prime}, \boldsymbol{p}_{2}+\boldsymbol{k}_{2}^{\prime}, \boldsymbol{p}_{3}+\boldsymbol{k}_{3}^{\prime}\right\} ; \boldsymbol{P}^{\prime}\right)\right]^{*} } \\
& \times \Psi_{\lambda}^{\left(\lambda_{1}, \lambda_{2}\right) \lambda_{3}}\left(\left\{\alpha_{i}\right\},\left\{\boldsymbol{p}_{1}-\boldsymbol{k}_{1}, \boldsymbol{p}_{2}-\boldsymbol{k}_{2}, \boldsymbol{p}_{3}-\boldsymbol{k}_{3}\right\} ; \boldsymbol{P}\right),
\end{aligned}
$$

where $\boldsymbol{k}_{i}\left(\boldsymbol{k}_{j}^{\prime}\right)$ is the sum of momenta delivered by the gluons to the quark line $i$ to the left (right) of the central cutting line. Obviously,

$$
\sum_{i} k_{i}+\sum_{j} \boldsymbol{k}_{j}^{\prime}=\boldsymbol{P}^{\prime}-\boldsymbol{P}
$$




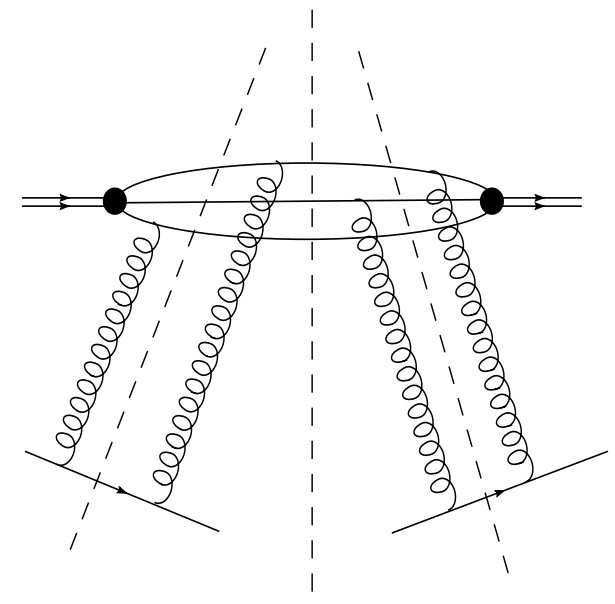

Figure 9: A contribution to the four gluon exchange in the scattering of a baryon on two independent quarks.

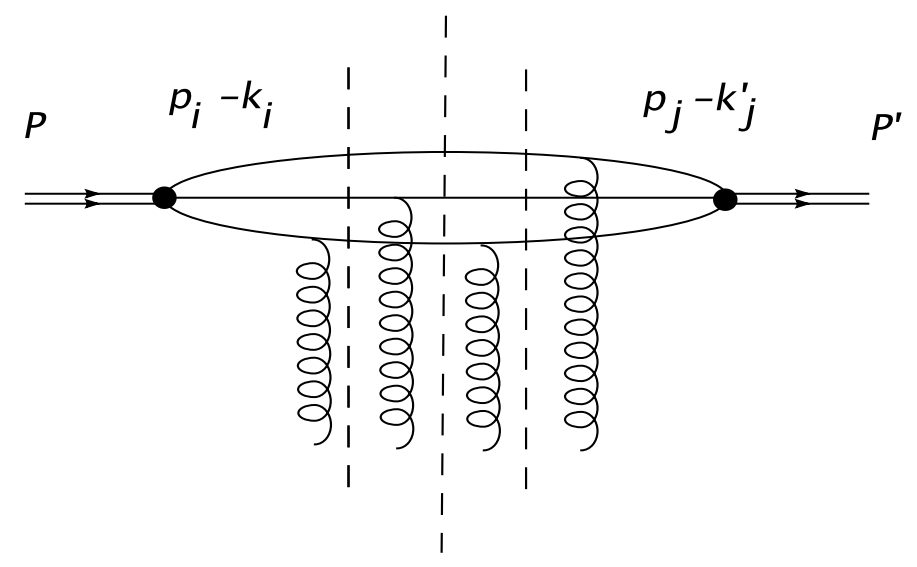

Figure 10: Example of a diagram defining the overlap function for a multiple discontinuity in Fig. 9. 


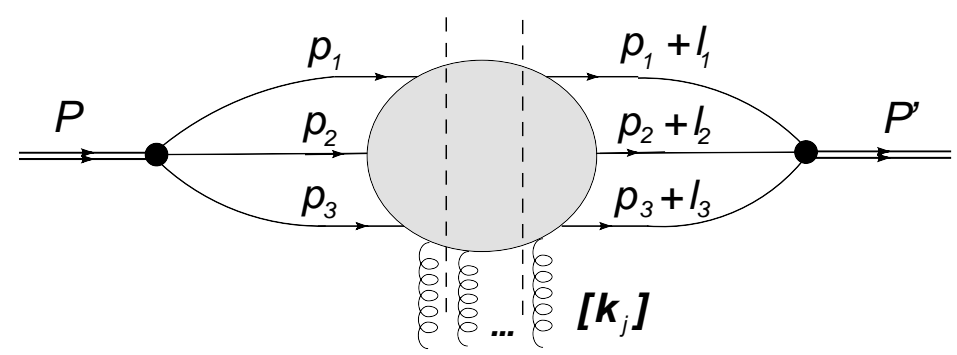

Figure 11: Baryon impact factor.

Since, as a consequence of the exponential form factor, all transverse momentum integrals are finite, we are allowed to shift, for each diagram separately, the loop momenta, such that to the right of the incoming baryon vertex, the momenta become $\boldsymbol{p}_{1}, \boldsymbol{p}_{2}$, and $\boldsymbol{p}_{3}$. At the outgoing baryon vertex the momenta are $\boldsymbol{p}_{1}+\boldsymbol{l}_{1}$, $\boldsymbol{p}_{2}+\boldsymbol{l}_{2}$, and $\boldsymbol{p}_{3}+\boldsymbol{l}_{3}$, where $\boldsymbol{l}_{i}$ is the sum of transverse momenta of all gluons coupled to the quark line $i$ (Fig. 10):

$$
\boldsymbol{l}_{i}=\sum_{j \in L_{i}}\left(\boldsymbol{k}_{j}+\boldsymbol{k}_{j}^{\prime}\right)
$$

In the general case the overlap function can be written as:

$$
\mathcal{F}^{\lambda \lambda^{\prime}}\left(\left\{\boldsymbol{l}_{i}\right\} ; \boldsymbol{P}, \boldsymbol{P}^{\prime}\right)=\sum_{\lambda_{1}, \lambda_{2}, \lambda_{3}} \int\left[d^{2} \boldsymbol{p}_{i}\right]\left[d \alpha_{i}\right]\left[\Psi_{\lambda^{\prime}}^{\left(\lambda_{1}, \lambda_{2}\right) \lambda_{3}}\left(\left\{\alpha_{i}\right\},\left\{\boldsymbol{p}_{i}+\boldsymbol{l}_{i}\right\} ; \boldsymbol{P}^{\prime}\right)\right]^{*} \Psi_{\lambda}^{\left(\lambda_{1}, \lambda_{2}\right) \lambda_{3}}\left(\left\{\alpha_{i}\right\},\left\{\boldsymbol{p}_{i}\right\} ; \boldsymbol{P}\right) .
$$

We now complete the definition of the baryonic impact factor (see Fig. 11). The general impact factor $\mathcal{I}_{A B}^{(N)}$ for the transition $A \rightarrow B$, with $N$ gluons being coupled in the eikonal approximation, is defined as

$$
\mathcal{I}_{A B}^{(N)}=\int \frac{d \beta_{1}}{2 \pi} \frac{d \beta_{2}}{2 \pi} \ldots \frac{d \beta_{N-1}}{2 \pi} \frac{q_{\mu_{1}} q_{\mu_{2}} \ldots q_{\mu_{N}}}{s} \operatorname{Disc}_{N-1} \mathcal{S}_{A B}^{\mu_{1} \mu_{2} \ldots \mu_{N}}
$$

where $\mathcal{S}_{A B}^{\mu_{1} \mu_{2} \ldots \mu_{N}}$ represents the amputated transition amplitude. In particular, for the elastic scattering of a single quark inside the baryon one obtains:

$$
\mathcal{I}_{q q}^{(N)}=\frac{1}{N_{c}} \operatorname{Tr}\left[t^{a_{N}} t^{a_{N-1}} \ldots t^{a_{1}}\right] I_{q q}^{(N)},
$$

where

$$
I_{q q}^{(N)}=(-i g)^{N} .
$$

The baryon impact factor for $N t$-channel gluons is then given by

$$
\mathcal{B}_{N}^{\lambda \lambda^{\prime}}\left(\left\{\boldsymbol{l}_{i}\right\} ; \boldsymbol{P}, \boldsymbol{P}^{\prime}\right)=I_{q q}^{(N)} \sum_{\text {diagrams }} \mathcal{F}^{\lambda \lambda^{\prime}}\left(\left\{\boldsymbol{l}_{i}\right\} ; \boldsymbol{P}, \boldsymbol{P}^{\prime}\right) \mathcal{C}_{N}(\text { diagram }),
$$

where the color factor reads:

$$
\mathcal{C}_{N}(\text { diagram })=\frac{\varepsilon^{\kappa_{1}^{\prime} \kappa_{2}^{\prime} \kappa_{3}^{\prime}} \varepsilon^{\kappa_{1} \kappa_{2} \kappa_{3}}}{3 !}\left[t^{a_{l}} t^{a_{l-1}} \ldots t^{a_{1}}\right]_{\kappa_{1}^{\prime} \kappa_{1}}\left[t^{b_{m}} t^{b_{m-1}} \ldots t^{b_{1}}\right]_{\kappa_{2}^{\prime} \kappa_{2}}\left[t^{c_{n}} t^{c_{n-1}} \ldots t^{c_{1}}\right]_{\kappa_{3}^{\prime} \kappa_{3}} .
$$

In (43) the sum extends over all diagrams, and the numbers $l, m$ and $n$ of gluons, that couple to quark lines 1, 2 and 3, take all possible values between 1 and $N$ with the constraint $l+m+n=N$. The overlap 


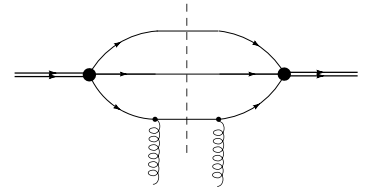

a)

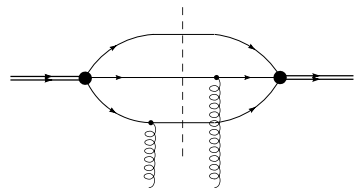

b)

Figure 12: Diagrams defining $B_{2 ; 0}$.

function $\mathcal{F}^{\lambda \lambda^{\prime}}\left(\left\{\boldsymbol{l}_{i}\right\} ; \boldsymbol{P}, \boldsymbol{P}^{\prime}\right)$ is evaluated for each diagram separately, and in each case it contains a global delta function of the transverse momenta:

$$
\mathcal{F}^{\lambda \lambda^{\prime}}\left(\left\{\boldsymbol{l}, \boldsymbol{l}^{\prime}\right\} ; \boldsymbol{P}, \boldsymbol{P}^{\prime}\right)=F^{\lambda \lambda^{\prime}}\left(\boldsymbol{l}_{1}, \boldsymbol{l}_{2}, \boldsymbol{l}_{3}\right) \delta^{(2)}\left(\sum_{i} \boldsymbol{l}_{i}+\boldsymbol{P}-\boldsymbol{P}^{\prime}\right) .
$$

We impose the normalization condition:

$$
\left.F^{\lambda \lambda^{\prime}}\left(\boldsymbol{l}_{1}, \boldsymbol{l}_{2}, \boldsymbol{l}_{3}\right)\right|_{\boldsymbol{l}_{1}=l_{2}=l_{3}=0}=\delta^{\lambda \lambda^{\prime}} .
$$

Correspondingly, we also extract a delta function from the impact factor:

$$
\mathcal{B}_{N}^{\lambda \lambda^{\prime}}\left(\left\{\boldsymbol{l}_{i}\right\} ; \boldsymbol{P}, \boldsymbol{P}^{\prime}\right)=B_{N}^{\lambda \lambda^{\prime}}\left(\left\{\boldsymbol{l}_{i}\right\}\right) \delta^{(2)}\left(\sum_{i} \boldsymbol{l}_{i}+\boldsymbol{P}-\boldsymbol{P}^{\prime}\right) .
$$

In the following we will restrict ourselves to the forward direction, $\boldsymbol{P}=\boldsymbol{P}^{\prime}=0$. Because of helicity conservation for the impact factor, we always have $\lambda=\lambda^{\prime}$, and we will drop the upper helicity labels, i.e. $F^{\lambda \lambda^{\prime}}\left(\boldsymbol{l}_{1}, \boldsymbol{l}_{2}, \boldsymbol{l}_{3}\right) \rightarrow F\left(\boldsymbol{l}_{1}, \boldsymbol{l}_{2}, \boldsymbol{l}_{3}\right)$ etc. We will go through the cases of $N=2, N=3$, and $N=4$ gluons. We therefore define, for fixed $N$, the functions $B_{N ; 0}\left(\left\{\boldsymbol{l}_{i}\right\}\right)$ projected on the $C$-even channel through

$$
\left.\mathcal{B}_{N}^{\lambda \lambda}\left(\left\{\boldsymbol{l}_{i}\right\} ; \boldsymbol{P}, \boldsymbol{P}^{\prime}\right)\right|_{\boldsymbol{P}=\boldsymbol{P}^{\prime}=0} ^{\mathrm{C}-\text { even }}=B_{N ; 0}\left(\left\{\boldsymbol{l}_{i}\right\}\right) \quad \delta^{(2)}\left(\sum \boldsymbol{l}_{i}\right)
$$

and analogously for the $C$-odd projections $\tilde{B}_{N ; 0}$.

In the remaining part of this section the main emphasis will be on the color structure of the impact factors, contained in Eq. (44). As the main result, we will find a decomposition into a sum of terms which, as it will be demonstrated in the subsequent section, stays invariant under evolution in rapidity. We stress that the results which follow are valid for an arbitrary overlap function $F\left(\boldsymbol{l}_{1}, \boldsymbol{l}_{2}, \boldsymbol{l}_{3}\right)$, i.e. they do not rely on a particular model of the baryon wave function, provided the baryon has the valence degrees of freedom of three quarks.

\subsection{Two gluons}

We begin with the two-gluon coupling which is $C$-even. All diagrams are proportional the color tensor $\delta^{a_{1} a_{2}}$, and it is suggestive to group them into three sets: in the first one, the two gluons couple to the quark pair (12), and quark 3 acts as a spectator (Fig. 12). In the second one the gluons couple to (13) and quark 2 acts as spectator etc. Inside each set, we have four terms. We thus find:

$$
B_{2 ; 0}\left(\boldsymbol{k}_{1}, \boldsymbol{k}_{2}\right)=\delta^{a_{1} a_{2}}\left[D_{2 ; 0}^{\{1,2\}}\left(\boldsymbol{k}_{1}, \boldsymbol{k}_{2}\right)+D_{2 ; 0}^{\{1,3\}}\left(\boldsymbol{k}_{1}, \boldsymbol{k}_{2}\right)+D_{2 ; 0}^{\{2,3\}}\left(\boldsymbol{k}_{1}, \boldsymbol{k}_{2}\right)\right],
$$




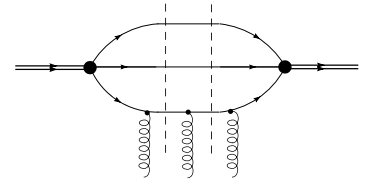

a)

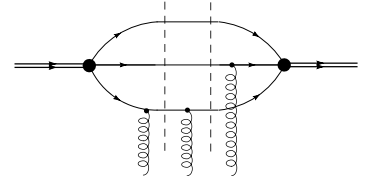

b)

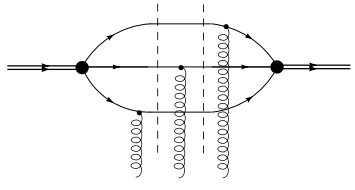

c)

Figure 13: Examples of diagrams defining $B_{3 ; 0}$ and $\tilde{B}_{3 ; 0}$.

with

$$
\begin{aligned}
& D_{2 ; 0}^{\{1,2\}}\left(\boldsymbol{k}_{1}, \boldsymbol{k}_{2}\right)=\frac{-g^{2}}{12}\left[F(\boldsymbol{k}, 0,0)+F(0, \boldsymbol{k}, 0)-F\left(\boldsymbol{k}_{1}, \boldsymbol{k}_{2}, 0\right)-F\left(\boldsymbol{k}_{2}, \boldsymbol{k}_{1}, 0\right)\right], \\
& D_{2 ; 0}^{\{1,3\}}\left(\boldsymbol{k}_{1}, \boldsymbol{k}_{2}\right)=\frac{-g^{2}}{12}\left[F(\boldsymbol{k}, 0,0)+F(0,0, \boldsymbol{k})-F\left(\boldsymbol{k}_{1}, 0, \boldsymbol{k}_{2}\right)-F\left(\boldsymbol{k}_{2}, 0, \boldsymbol{k}_{1}\right)\right], \\
& D_{2 ; 0}^{\{2,3\}}\left(\boldsymbol{k}_{2}, \boldsymbol{k}_{3}\right)=\frac{-g^{2}}{12}\left[F(0, \boldsymbol{k}, 0)+F(0,0, \boldsymbol{k})-F\left(0, \boldsymbol{k}_{1}, \boldsymbol{k}_{2}\right)-F\left(0, \boldsymbol{k}_{2}, \boldsymbol{k}_{1}\right)\right],
\end{aligned}
$$

where $\boldsymbol{k}_{1}, \boldsymbol{k}_{2}$ denote the gluon momenta and $\boldsymbol{k}=\boldsymbol{k}_{1}+\boldsymbol{k}_{2}$. On the r.h.s. of (50)-(52), the momentum arguments of the $F$ functions indicate which diagrams they represent: in the first (second) term of (50), both gluons couple to quark line 1 (2). In the third term, the first gluon couples to line 1, the second to line 2, and so on. The relative signs arise from the color structure. As a striking result, on the r.h.s. of (50) - (52), in each line the four terms have the same structure as the impact factor of the photon. In particular, each set satisfies the Ward identities, i.e. it vanishes as any of its momenta goes to zero. Since the pair of scattering quarks $\{i, j\}$ is in a color anti-triplet state, one might, at first sight, interpret this set as the elastic scattering of an 'anti-triplet dipole'. However, it is important to stress that these three dipole-like components $D_{2 ; 0}^{\{i, j\}}$, are not independent from each other: the diagrams where two gluons couple to the same quark line, say, line 3 in Fig. 12a, contribute both to the pair (13) and (23). In this sense, one better views these quark pairs as 'anti-triplets inside the baryon'. Also, these configurations where one quark pair interacts whereas the third quark remains a spectator, should not simply be viewed as 'diquark states': in transverse coordinate space, the spectator quark can be far away from the quark pair (see the discussion in Section 7). One should also add that the normalization of the dipole-like components $D_{2 ; 0}^{\{i, j\}}$ of the baryon impact factor is exactly $1 / 2$ of the normalization of the genuine color dipole impact factor. At the two-gluon level, our results coincide with results of Ref. [27].

If, instead of our model for the baryonic impact factor, we would have used a completely symmetric baryon form-factor $F^{(s)}$ (which does not discriminate between $u$ and $d$ quarks) we would have arrived at a familiar result [28]:

$$
B_{2 ; 0}^{(s)}\left(\boldsymbol{k}_{1}, \boldsymbol{k}_{2}\right)=\frac{-g^{2}}{2} \delta^{a_{1} a_{2}}\left[F^{(s)}(\boldsymbol{k}, 0,0)-F^{(s)}\left(\boldsymbol{k}_{1}, \boldsymbol{k}_{2}, 0\right)\right]
$$

\subsection{Three gluons}

In the case of three gluons (Fig. 13) we have to distinguish between even and odd $C$ parity: in the color trace Eq. (44) we find both color tensors, $f^{a_{1} a_{2} a_{3}}$ and $d^{a_{1} a_{2} a_{3}}$. The first one belongs to even (Pomeron), the second to odd (odderon) $C$ parity. 
The $C$-odd baryon impact factor reads $[16,29,30]$

$$
\tilde{B}_{3 ; 0}\left(\boldsymbol{k}_{1}, \boldsymbol{k}_{2}, \boldsymbol{k}_{3}\right)=d^{a_{1} a_{2} a_{3}} E_{3 ; 0}\left(\boldsymbol{k}_{1}, \boldsymbol{k}_{2}, \boldsymbol{k}_{3}\right),
$$

where

$$
E_{3 ; 0}\left(\boldsymbol{k}_{1}, \boldsymbol{k}_{2}, \boldsymbol{k}_{3}\right)=\frac{i g^{3}}{24} \sum_{\sigma}\left[2 F^{\sigma}\left(\boldsymbol{k}_{1}, \boldsymbol{k}_{2}, \boldsymbol{k}_{3}\right)-\sum_{i=1}^{3} F^{\sigma}\left(\boldsymbol{k}_{i}, \boldsymbol{k}-\boldsymbol{k}_{i}, 0\right)+F^{\sigma}(\boldsymbol{k}, 0,0)\right],
$$

and $F^{\sigma}$ denotes the $F$ functions, with its arguments being permuted by the permutation $\sigma$ :

$$
F^{\sigma}\left(\boldsymbol{l}_{1}, \boldsymbol{l}_{2}, \boldsymbol{l}_{3}\right)=F\left(\boldsymbol{l}_{\sigma(1)}, \boldsymbol{l}_{\sigma(2)}, \boldsymbol{l}_{\sigma(3)}\right) .
$$

In $E_{3 ; 0}$ the $t$-channel three gluon state is Bose symmetric, that is

$$
E_{3 ; 0}\left(\boldsymbol{k}_{\sigma(1)}, \boldsymbol{k}_{\sigma(2)}, \boldsymbol{k}_{\sigma(3)}\right)=E_{3 ; 0}\left(\boldsymbol{k}_{1}, \boldsymbol{k}_{2}, \boldsymbol{k}_{3}\right)
$$

for any permutation $\sigma$, and it obeys the Ward identities:

$$
E_{3 ; 0}\left(\boldsymbol{k}_{1}, \boldsymbol{k}_{2}, \boldsymbol{k}_{3}\right)=0 \text { for any } \boldsymbol{k}_{j} \rightarrow 0 \text {. }
$$

On the r.h.s. of Eq. (55), the momentum structure of first term indicates that the three gluons couple to three quarks. The second and third term play the role of subtractions. This leads to the interpretation that, in this piece of the baryonic impact factor, in contrast to the structure found previously for 2 gluons, all three quarks participate in the interaction. Since each of the three gluon has negative $C$ parity, this $t$-channel belongs to the $C=-$ (odderon) state.

For a completely symmetric model for the baryon form-factor expression (55), again, reduces to a known result [29]:

$$
\tilde{B}_{3 ; 0}^{(s)}\left(\boldsymbol{k}_{1}, \boldsymbol{k}_{2}, \boldsymbol{k}_{3}\right)=\frac{i g^{3}}{4} d^{a_{1} a_{2} a_{3}}\left[2 F^{(s)}\left(\boldsymbol{k}_{1}, \boldsymbol{k}_{2}, \boldsymbol{k}_{3}\right)-\sum_{i=1}^{3} F^{(s)}\left(\boldsymbol{k}_{i}, \boldsymbol{k}-\boldsymbol{k}_{i}, 0\right)+F^{(s)}(\boldsymbol{k}, 0,0)\right] .
$$

Next we turn to the terms proportional to $f^{a_{1} a_{2} a_{3}}$ which turn out to belong to even $C$. They can be grouped in the same 'dipole-like' form as in (49):

$$
B_{3 ; 0}\left(\boldsymbol{k}_{1}, \boldsymbol{k}_{2}, \boldsymbol{k}_{3}\right)=D_{3 ; 0}^{\{1,2\}}\left(\boldsymbol{k}_{1}, \boldsymbol{k}_{2}, \boldsymbol{k}_{3}\right)+D_{3 ; 0}^{\{1,3\}}\left(\boldsymbol{k}_{1}, \boldsymbol{k}_{2}, \boldsymbol{k}_{3}\right)+D_{3 ; 0}^{\{2,3\}}\left(\boldsymbol{k}_{1}, \boldsymbol{k}_{2}, \boldsymbol{k}_{3}\right),
$$

where the dipole-like components have the structure known from the photon case,

$$
D_{3 ; 0}^{\{i, j\}}\left(\boldsymbol{k}_{1}, \boldsymbol{k}_{2}, \boldsymbol{k}_{3}\right)=\frac{1}{2} g f^{a_{1} a_{2} a_{3}}\left[D_{2 ; 0}^{\{i, j\}}\left(\boldsymbol{k}_{1}+\boldsymbol{k}_{2}, \boldsymbol{k}_{3}\right)-D_{2 ; 0}^{\{i, j\}}\left(\boldsymbol{k}_{1}+\boldsymbol{k}_{3}, \boldsymbol{k}_{2}\right)+D_{2 ; 0}^{\{i, j\}}\left(\boldsymbol{k}_{2}+\boldsymbol{k}_{3}, \boldsymbol{k}_{1}\right)\right] .
$$

As in the photon case, the argument structure indicates the beginning of the reggeization of the gluons: for example, in the first term, the first two gluons with momenta $\boldsymbol{k}_{1}$ and $\boldsymbol{k}_{2}$ 'collapse' into a single reggeized gluon with momentum $\boldsymbol{k}_{1}+\boldsymbol{k}_{2}$. The $t$-channel system thus consists of two reggeized gluons only and hence belongs to $C$-even. In the next section we will show that this structure is preserved in the rapidity evolution.

In the following it will be convenient to use a shorthand notation by writing, instead of $D_{2 ; 0}^{\{i, j\}}\left(\boldsymbol{k}_{1}+\boldsymbol{k}_{2}, \boldsymbol{k}_{3}\right)$, simply $D_{2 ; 0}^{\{i, j\}}(12,3)$ etc. 


\subsection{Four gluons}

In the case of four gluons (Fig. 14) the color trace (44) contains $f f, d d, f d$, and $\delta \delta$ color tensor structures. Beginning with the $f d$ pieces, we find that they can be expressed in terms of the $E$-function (55) which we have obtained for the odderon channel:

$$
\begin{aligned}
\tilde{B}_{4 ; 0}(1,2,3,4) & = \\
& \frac{g}{2}\left(f^{a_{1} a_{2} b} d^{b a_{3} a_{4}} E_{3 ; 0}(12,3,4)+f^{a_{1} a_{3} b} d^{b a_{2} a_{4}} E_{3 ; 0}(13,2,4)+f^{a_{1} a_{4} b} d^{b a_{2} a_{3}} E_{3 ; 0}(14,2,3)\right. \\
& \left.+f^{a_{2} a_{3} b} d^{b a_{1} a_{4}} E_{3 ; 0}(23,1,4)+f^{a_{2} a_{4} b} d^{b a_{1} a_{3}} E_{3 ; 0}(24,1,3)+f^{a_{3} a_{4} b} d^{b a_{1} a_{2}} E_{3 ; 0}(34,1,2)\right) .
\end{aligned}
$$

We then interpret this contribution as the odderon configuration with one reggeizing gluon. It agrees with the result first found by C. Ewerz [16].

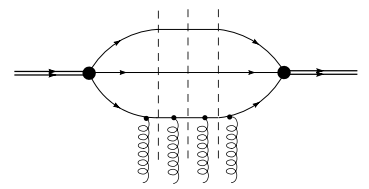

a)

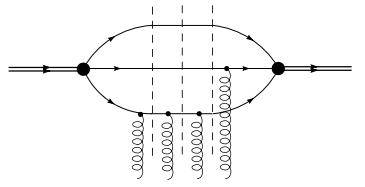

b)

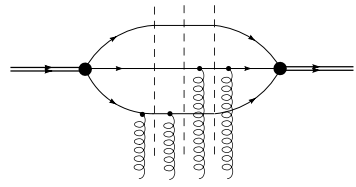

c)

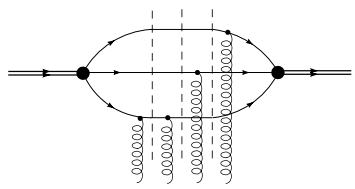

d)

Figure 14: Examples of diagrams defining $B_{4 ; 0}$ and $\tilde{B}_{4 ; 0}$.

Next the $f f, d d$, and $\delta \delta$ terms. We find, in addition to a set of pieces which have the same structure as in the photon case, a new structure, $Q_{4 ; 0}$. In detail:

$$
B_{4 ; 0}(1,2,3,4)=D_{4 ; 0}^{\{1,2\}}(1,2,3,4)+D_{4 ; 0}^{\{1,3\}}(1,2,3,4)+D_{4 ; 0}^{\{2,3\}}(1,2,3,4)+Q_{4 ; 0}(1,2,3,4) .
$$

Here the first three terms are dipole-like, and they follow the reggeization pattern found for the photon scattering:

$$
\begin{aligned}
D_{4 ; 0}^{\{i, j\}}(1,2,3,4)= & -g^{2}\left\{d^{a_{1} a_{2} a_{3} a_{4}}\left[D_{2 ; 0}^{\{i, j\}}(123,4)+D_{2 ; 0}^{\{i, j\}}(234,1)-D_{2 ; 0}^{\{i, j\}}(14,23)\right]\right. \\
& \left.+d^{a_{1} a_{2} a_{4} a_{3}}\left[D_{2 ; 0}^{\{i, j\}}(124,3)+D_{2 ; 0}^{\{i, j\}}(134,2)-D_{2 ; 0}^{\{i, j\}}(12,34)-D_{2 ; 0}^{\{i, j\}}(13,24)\right]\right\}
\end{aligned}
$$

with the color tensor

$$
d^{a_{1} a_{2} a_{3} a_{4}}=\frac{\delta^{a_{1} a_{2}} \delta^{a_{3} a_{4}}}{2 N_{c}}+\frac{d^{a_{1} a_{2} b} d^{b a_{3} a_{4}}}{4}-\frac{f^{a_{1} a_{2} b} f^{b a_{3} a_{4}}}{4} .
$$

In the next section we will study the rapidity evolution of these terms, and we will confirm that they follow the photon impact factor to all orders.

The new structure which has no analogue in the case of the photon looks as follows:

$$
\begin{aligned}
Q_{4 ; 0}(1,2,3,4)= & \frac{-i g}{2}\left[d^{a_{1} a_{2} b} d^{b a_{3} a_{4}}-\frac{1}{3} \delta^{a_{1} a_{2}} \delta^{a_{3} a_{4}}\right]\left[E_{3 ; 0}(12,3,4)+E_{3 ; 0}(34,1,2)\right]+ \\
& \frac{-i g}{2}\left[d^{a_{1} a_{3} b} d^{b a_{2} a_{4}}-\frac{1}{3} \delta^{a_{1} a_{3}} \delta^{a_{2} a_{4}}\right]\left[E_{3 ; 0}(13,2,4)+E_{3 ; 0}(24,1,3)\right]+ \\
& \frac{-i g}{2}\left[d^{a_{1} a_{4} b} d^{b a_{2} a_{3}}-\frac{1}{3} \delta^{a_{1} a_{4}} \delta^{a_{2} a_{3}}\right]\left[E_{3 ; 0}(14,2,3)+E_{3 ; 0}(23,1,4)\right] .
\end{aligned}
$$


The function $E$ is the same as in the odderon case, and, in particular, all three quarks participate in the interaction. The $t$-channel state which couples to $Q_{4 ; 0}$ is Bose symmetric

$$
Q_{4 ; 0}(\sigma(1), \sigma(2), \sigma(3), \sigma(4))=Q_{4 ; 0}(1,2,3,4)
$$

for any permutation $\sigma$, and it is gauge invariant:

$$
Q_{4 ; 0}\left(\boldsymbol{k}_{1}, \boldsymbol{k}_{2}, \boldsymbol{k}_{3}, \boldsymbol{k}_{4}\right)=0 \text { for any } \boldsymbol{k}_{j} \rightarrow 0
$$

This property may be proven using the identity for color tensors valid for $N_{c}=3$ :

$$
d^{a_{1} a_{2} b} d^{b a_{3} a_{4}}+d^{a_{1} a_{3} b} d^{b a_{2} a_{4}}+d^{a_{1} a_{4} b} d^{b a_{2} a_{3}}=\frac{1}{3}\left(\delta^{a_{1} a_{2}} \delta^{a_{3} a_{4}}+\delta^{a_{1} a_{3}} \delta^{a_{2} a_{4}}+\delta^{a_{1} a_{4}} \delta^{a_{2} a_{3}}\right) .
$$

The analysis in the following section will show that this novel piece of the baryon impact factor couples a three-gluon $t$-channel configuration in which one of the reggeized gluons is an even-signature $d$-Reggeon. The overall $C$ parity therefore is positive.

\section{$5 \quad$ Integral evolution equations}

In this section we study higher order corrections in the (generalized) leading logarithmic (log $s$ ) approximation. The all-order sum of these terms will be represented by integral equations [14, 15], written for Mellin moments of the multiple discontinuities with respect to the energy $s$. In our notation the dependence of the amplitudes $B_{N}$ and $\tilde{B}_{N}$ (and also $D_{N}, E_{N}$ and $Q_{N}$ ) on the Mellin variable $\omega$ is implicit.

Let us begin with the $C$-odd configurations. In the case of three gluons, the impact factor $E_{3 ; 0}$ is simply replaced by the Green's function function $E_{3}$, which satisfies the BKP equation for three odd signature Reggeons, with the initial condition given by $E_{3 ; 0}$ :

$$
\left(\omega-\sum_{i} \beta\left(\boldsymbol{k}_{i}\right)\right) E_{3}=E_{3 ; 0}+\sum_{(r, s)} K_{2 \rightarrow 2}(r, s) \otimes E_{3},
$$

where $K_{2 \rightarrow 2}$ is the real emission part of the BFKL kernel, and the odderon state with the full color structure reads

$$
\tilde{B}_{3}(1,2,3)=d^{a_{1} a_{2} a_{3}} E_{3}(1,2,3) .
$$

The four gluon case has been studied in [16], and we simply quote the solution:

$$
\begin{aligned}
\tilde{B}_{4}(1,2,3,4) & = \\
& \frac{g}{2}\left[f^{a_{1} a_{2} b} d^{b a_{3} a_{4}} E_{3}(12,3,4)+f^{a_{1} a_{3} b} d^{b a_{2} a_{4}} E_{3}(13,2,4)+f^{a_{1} a_{4} b} d^{b a_{2} a_{3}} E_{3}(14,2,3)\right. \\
& \left.+f^{a_{2} a_{3} b} d^{b a_{1} a_{4}} E_{3}(23,1,4)+f^{a_{2} a_{4} b} d^{b a_{1} a_{3}} E_{3}(24,1,3)+f^{a_{3} a_{4} b} d^{b a_{1} a_{2}} E_{3}(34,1,2)\right] .
\end{aligned}
$$

where the function $E_{3}$ has been defined before in (70). Clearly, the solution is saturated by a reggeizing contribution: in each term, one of the three $f$-Reggeons splits into two elementary gluons. 
We now turn to the $C$-even contributions. The integral equations for the multiple discontinuities read (up to four gluons):

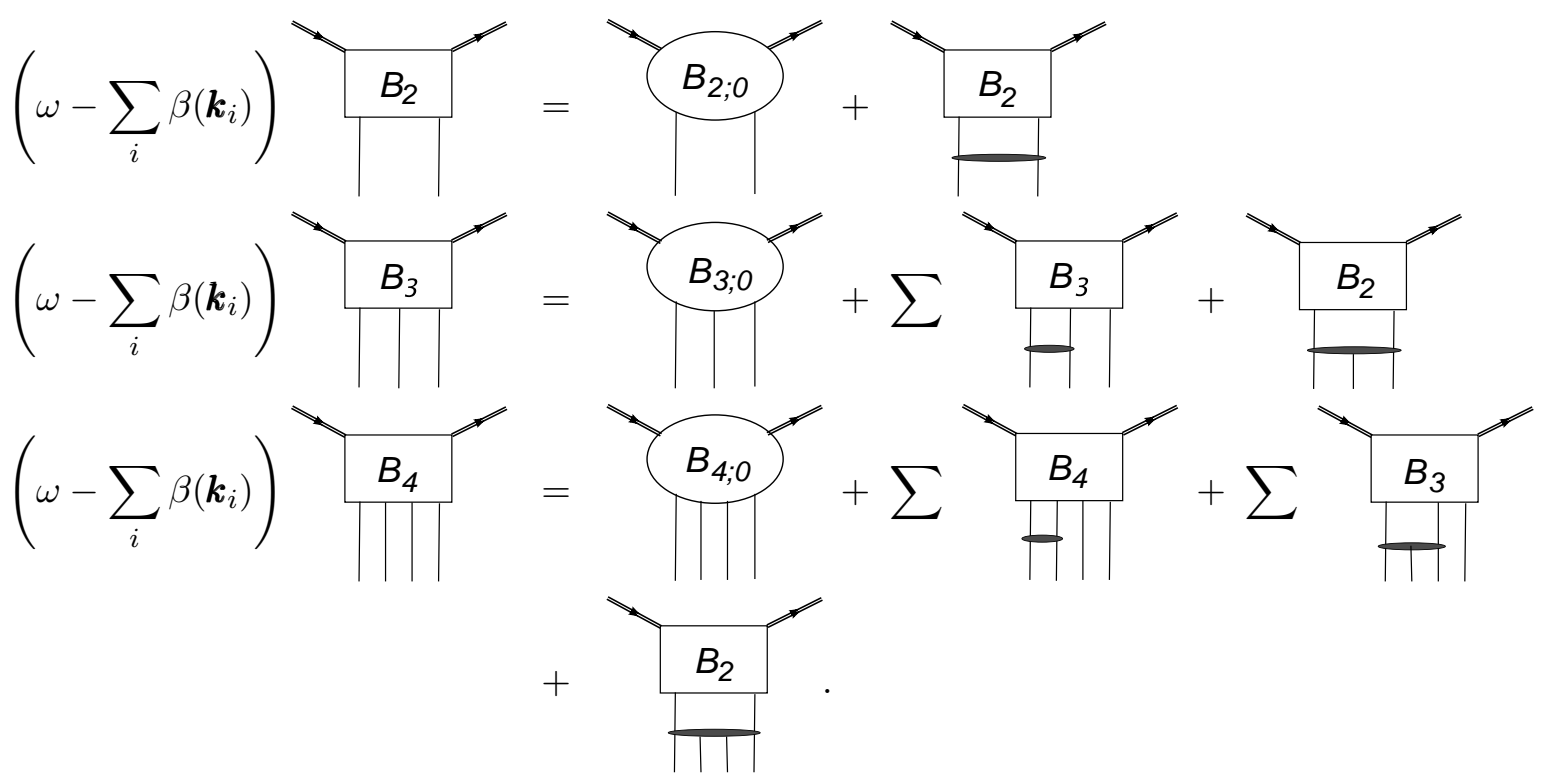

The integral kernels driving $2 \rightarrow 2,3,4, \ldots$ Reggeon transitions, that appear in the above equations include the color structure, and they are defined in Ref. [14,15]. The gluon Regge trajectory $\beta(\boldsymbol{k})$ will be specified below. The case of two gluons is the simplest one: $B_{2}$ satisfies the BFKL equation. According to the structure of the inhomogeneous term in Eq. (49), $B_{2}$ can be written as the sum of three terms $D_{2}^{\{i, j\}}$,

$$
B_{2}(1,2)=\delta^{a_{1} a_{2}}\left[D_{2}^{\{1,2\}}(1,2)+D_{2}^{\{1,3\}}(1,2)+D_{2}^{\{2,3\}}(1,2)\right],
$$

with

$$
\left(\omega-\sum_{i=1}^{2} \beta\left(\boldsymbol{k}_{i}\right)\right) D_{2}^{\{i, j\}}=D_{2 ; 0}^{\{i, j\}}+K_{2 \rightarrow 2} \otimes D_{2}^{\{i, j\}} .
$$

In the case of three gluons, $B_{3}$ is given by the sum of three dipole-like components (cf. (60)):

$$
B_{3}(1,2,3)=D_{3}^{\{1,2\}}(1,2,3)+D_{3}^{\{1,3\}}(1,2,3)+D_{3}^{\{2,3\}}(1,2,3),
$$

where each term consists of three reggeizing pieces:

$$
D_{3}^{\{i, j\}}(1,2,3)=\frac{1}{2} g f^{a_{1} a_{2} a_{3}}\left[D_{2}^{\{i, j\}}(12,3)-D_{2}^{\{i, j\}}(13,2)+D_{2}^{\{i, j\}}(23,1)\right] .
$$

This structure coincides with the photon case.

The case of $B_{4}$ is more complex. Following our result for the baryon impact factor in Eq. (63) we decompose $B_{4}$ in the following way:

$$
B_{4}(1,2,3,4)=D_{4}^{\{1,2\}}(1,2,3,4)+D_{4}^{\{1,3\}}(1,2,3,4)+D_{4}^{\{2,3\}}(1,2,3,4)+Q_{4}(1,2,3,4) .
$$

For the dipole-like pieces $D_{4}^{\{i, j\}}$ we make use of the 'reduction procedure' developed for the photon case. Namely we decompose each $D_{4}^{\{i, j\}}$ into a reggeizing and an irreducible contributions

$$
D_{4}^{\{i, j\}}(1,2,3,4)=D_{4}^{\{i, j\} ; R}(1,2,3,4)+D_{4}^{\{i, j\} ; I}(1,2,3,4),
$$


with the reggeizing contribution given by

$$
\begin{aligned}
D_{4}^{\{i, j\} ; R}(1,2,3,4)= & -g^{2}\left\{d^{a_{1} a_{2} a_{3} a_{4}}\left[D_{2}^{\{i, j\}}(123,4)+D_{2}^{\{i, j\}}(234,1)-D_{2}^{\{i, j\}}(14,23)\right]\right. \\
& \left.+d^{a_{1} a_{2} a_{4} a_{3}}\left[D_{2}^{\{i, j\}}(124,3)+D_{2}^{\{i, j\}}(134,2)-D_{2}^{\{i, j\}}(12,34)-D_{2}^{\{i, j\}}(13,24)\right]\right\} .
\end{aligned}
$$

The reggeizing contributions are simple BFKL ladders with one reggeizing gluon splitting into three gluons or both reggeized gluons each splitting into two gluons. The irreducible contribution, containing the $2 \rightarrow 4$ Reggeon transition vertex, is illustrated in Fig. 15.

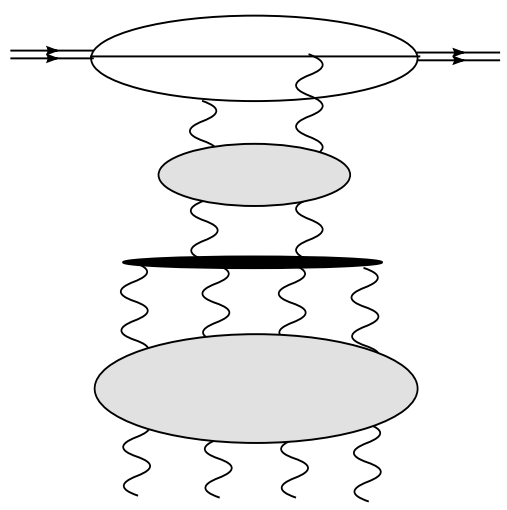

Figure 15: The component $D_{4}^{\{i, j\} ; I}(1,2,3,4)$.

These results provide further evidence that the 'dipole-like' pieces of the baryonic impact factor really behave in exactly the same way as the color dipole photon impact factor. In particular, if we would apply the large $N_{c}$ limit to the gluon evolution below the impact factor (which, of course, would be inconsistent with our finite- $N_{c}$ baryon), the four gluon system below the $2 \rightarrow 4$ transition vertex would split into two non-interacting BFKL ladders, and we would arrive at the first iteration of the BK equation.

After subtracting, from $B_{4}(1,2,3,4)$ in $(80)$, these dipole-like contributions of the baryon we are left with $Q_{4}$. As $Q_{4}$ appears at level of four gluons, its evolution equation has simply the BKP form:

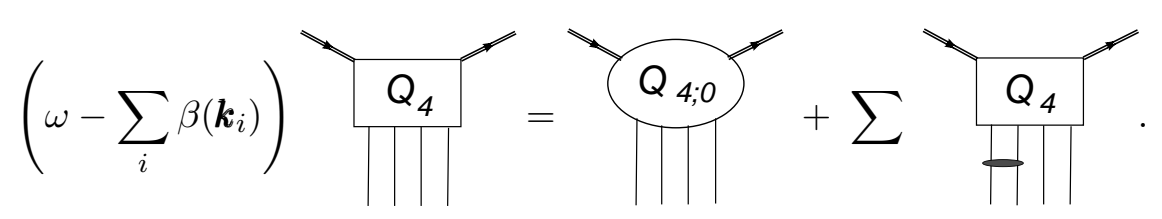

Making use of the experience with $D_{4}$, we decompose the amplitude $Q_{4}$ into a reggeizing piece $Q_{4}^{R}$ and an irreducible contribution $Q_{4}^{I}$ :

$$
Q_{4}(1,2,3,4)=Q_{4}^{R}(1,2,3,4)+Q_{4}^{I}(1,2,3,4) .
$$

Going through steps similar to the ones outlined in $[14,15]$ we find that the reggeizing pieces $Q_{4}^{R}$ preserve 


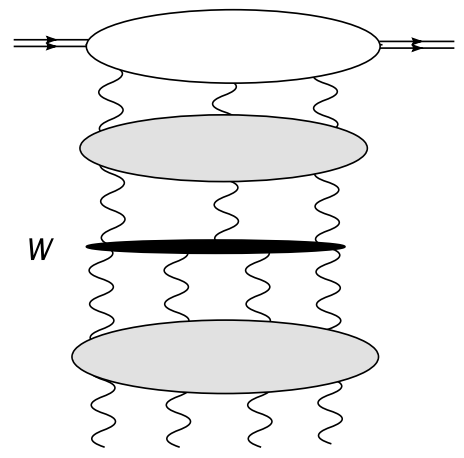

Figure 16: The new $3 \rightarrow 4$ transition vertex $W$.

the structure of $Q_{4 ; 0}$ :

$$
\begin{aligned}
Q_{4}^{R}(1,2,3,4)= & \frac{-i g}{2}\left[d^{a_{1} a_{2} b} d^{b a_{3} a_{4}}-\frac{1}{3} \delta^{a_{1} a_{2}} \delta^{a_{3} a_{4}}\right]\left[E_{3}(12,3,4)+E_{3}(34,1,2)\right]+ \\
& \frac{-i g}{2}\left[d^{a_{1} a_{3} b} d^{b a_{2} a_{4}}-\frac{1}{3} \delta^{a_{1} a_{3}} \delta^{a_{2} a_{4}}\right]\left[E_{3}(13,2,4)+E_{3}(24,1,3)\right]+ \\
& \frac{-i g}{2}\left[d^{a_{1} a_{4} b} d^{b a_{2} a_{3}}-\frac{1}{3} \delta^{a_{1} a_{4}} \delta^{a_{2} a_{3}}\right]\left[E_{3}(14,2,3)+E_{3}(23,1,4)\right] .
\end{aligned}
$$

As seen from the color and momentum structure, the three gluon state coupling to $Q_{4 ; 0}$ consists of three reggeized gluons, one of which is in a $d$ state and decays into two elementary gluons (the pieces proportional to color tensors $\delta \delta$ play the role of subtractions; in particular, they are needed in order to satisfy the Ward identities). This state, consisting of two odd signature $f$-Reggeon and one even signature $d$-Reggeon, belongs to even $C$, i.e. to the Pomeron channel.

The remaining piece, $Q_{4}^{I}$, contains a new transition vertex. We illustrate this contribution in Fig. 16. This vertex describes the transition from the three Reggeon state consisting of two $f$ and one $d$ Reggeon to four $f$ Reggeons. In more detail, the vertex may be completely decomposed into non-connected pieces of two types: (i) the incoming $d$ Reggeon together with one of the $f$ Reggeons makes a transition into three $f$ Reggeons whereas the remaining $f$ Reggeon acts as a (t-channel) spectator, and (ii): two $f$ Reggeons interact via the BFKL kernel and the $d$ Reggeon splits into two $f$ Reggeons. The explicit functional form of the vertex $W$, acting on the three Reggeon state $\phi_{3}$ is the following:

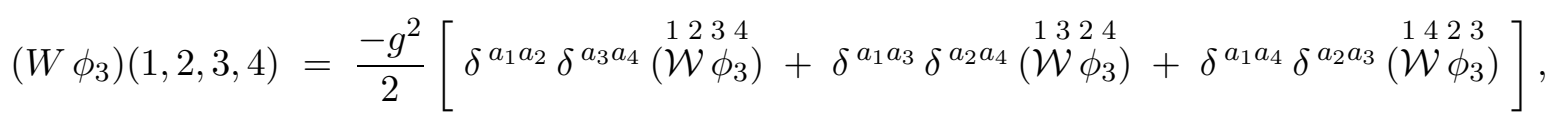

where

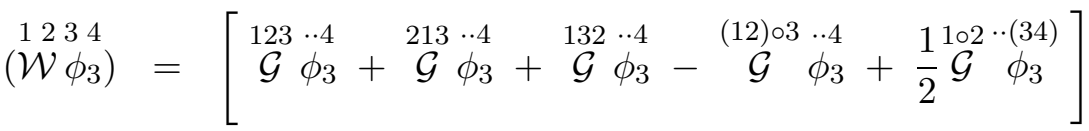

$$
\begin{aligned}
& +[3 \leftrightarrow 4]+[1 \leftrightarrow 3,2 \leftrightarrow 4]+[1 \leftrightarrow 4,2 \leftrightarrow 3] .
\end{aligned}
$$

Let us stress that this vertex acts on a completely symmetric function $\phi_{3}$ with three arguments, which inherits its structure from $E_{3}$. This vertex is closely related to a $3 \rightarrow 4$ vertex found in Ref. [31] in an analysis of jet production amplitudes at small $x$. The symbol $\stackrel{123}{\mathcal{G}}$ denotes the integral operator $G(1,2,3)$, 
introduced first in [14] and further investigated in [32]. It acts on a two gluon amplitude, $\phi_{2}$, and describes a transition to three gluons. It consists of two pieces:

$$
G(1,2,3)=G_{1}(1,2,3)+G_{2}(1,2,3)
$$

where the first one contains $s$-channel gluons ('connected part'), the second one reggeizing pieces ('disconnected part'):

$$
\begin{gathered}
\left(G_{1} \phi_{2}\right)\left(\boldsymbol{k}_{1}, \boldsymbol{k}_{2}, \boldsymbol{k}_{3}\right)= \\
\int \frac{d^{2} l}{(2 \pi)^{3}}\left(\frac{\left(\boldsymbol{k}_{2}+\boldsymbol{k}_{3}\right)^{2} \boldsymbol{l}^{2}}{\left(\boldsymbol{l}-\boldsymbol{k}_{1}\right)^{2}}+\frac{\left(\boldsymbol{k}_{1}+\boldsymbol{k}_{2}\right)^{2}(\boldsymbol{k}-\boldsymbol{l})^{2}}{\left(\boldsymbol{k}-\boldsymbol{l}-\boldsymbol{k}_{3}\right)^{2}}-\frac{\boldsymbol{k}_{2}^{2}(\boldsymbol{k}-\boldsymbol{l})^{2} \boldsymbol{l}^{2}}{\left(\boldsymbol{l}-\boldsymbol{k}_{1}\right)^{2}\left(\boldsymbol{k}-\boldsymbol{l}-\boldsymbol{k}_{3}\right)^{2}}-\boldsymbol{k}^{2}\right) \phi_{2}(\boldsymbol{l}, \boldsymbol{k}-\boldsymbol{l}),
\end{gathered}
$$

and

$$
\begin{gathered}
N_{c} g^{2}\left(G_{2} \phi_{2}\right)\left(\boldsymbol{k}_{1}, \boldsymbol{k}_{2}, \boldsymbol{k}_{3}\right)= \\
\int \frac{d^{2} l}{(2 \pi)^{3}} \boldsymbol{l}^{2}(\boldsymbol{k}-\boldsymbol{l})^{2}\left\{\left[\beta\left(\boldsymbol{k}_{2}+\boldsymbol{k}_{3}\right)-\beta\left(\boldsymbol{k}_{2}\right)\right](2 \pi)^{3} \delta^{(2)}\left(\boldsymbol{l}-\boldsymbol{k}_{1}\right)\right. \\
\left.+\left[\beta\left(\boldsymbol{k}_{1}+\boldsymbol{k}_{2}\right)-\beta\left(\boldsymbol{k}_{2}\right)\right](2 \pi)^{3} \delta^{(2)}\left(\boldsymbol{l}-\boldsymbol{k}_{3}\right)\right\} \phi_{2}(\boldsymbol{l}, \boldsymbol{k}-\boldsymbol{l}),
\end{gathered}
$$

with the gluon trajectory function

$$
\beta\left(\boldsymbol{k}_{i}\right)=-N_{c} g^{2} \int \frac{d^{2} \boldsymbol{l}}{(2 \pi)^{3}} \frac{\boldsymbol{k}_{i}^{2}}{\boldsymbol{l}^{2}+\left(\boldsymbol{k}_{i}-\boldsymbol{l}\right)^{2}} \frac{1}{\left(\boldsymbol{k}_{i}-\boldsymbol{l}\right)^{2}},
$$

and $\boldsymbol{k}=\boldsymbol{k}_{1}+\boldsymbol{k}_{2}+\boldsymbol{k}_{3}$. In (87) we have used a short-hand notation for the argument structure introduced in Ref. [31]: in the first term, $\stackrel{123}{\mathcal{G}} \phi_{3}, \phi_{3}$ is the three gluon amplitude above the vertex $W$ where the rightmost Reggeon (momentum $\boldsymbol{k}_{4}$ ) is a spectator, and the $G$ operator acts on the two left Reggeons, turning them into the three gluons with momenta $\boldsymbol{k}_{1}, \boldsymbol{k}_{2}$, and $\boldsymbol{k}_{3}$. In the fourth term, $\underset{\mathcal{G}}{(12) \circ 3} \phi_{3}$, Reggeon 4 is, again, a spectator, and the $G$ operator (with zero momentum in the second outgoing gluon) equals the BFKL kernel acting on the two leftmost gluons inside $\phi_{3}$ : after this BFKL interaction the leftmost gluon splits into two gluons with momenta $\boldsymbol{k}_{1}$ and $\boldsymbol{k}_{2}$, and the other one carries momentum $\boldsymbol{k}_{3}$. Finally, in the last term, ${ }_{\mathcal{G}}^{102 \cdot(34)} \phi_{3}$, the rightmost spectator now splits into two gluons with momenta $\boldsymbol{k}_{3}$ and $\boldsymbol{k}_{4}$, and the $G$ operator, like in the previous term, equals the BFKL operator with outgoing momenta $\boldsymbol{k}_{1}$ and $\boldsymbol{k}_{2}$.

The full vertex $W$ in (86) is gauge invariant, infra-red finite and Bose symmetric. As the vertex is expressed in terms of the function $\mathcal{G}$, it is also Möbius invariant [32]. Finally, there is no violation of signature conservation: the incoming three Reggeon state, consisting of one $d$-Reggeon and two $f$-Reggeons, has even signature; the same holds for the outgoing four Reggeon state (four $f$-Reggeons).

As a result, the baryonic impact factor introduces a new contribution to the Pomeron channel which has no analogue in the photon dipole factor. 


\section{Baryon wave functions in the coordinate space}

The baryon wave function in transverse position space may be easily obtained by the Fourier transform:

$$
\tilde{\Psi}_{\lambda}^{\left(\lambda_{1}, \lambda_{2}\right) \lambda_{3}}\left(\left\{\alpha_{i}\right\},\left\{\boldsymbol{r}_{i}\right\}, \boldsymbol{P}\right)=\int \frac{d^{2} p_{1}}{2 \pi} \frac{d^{2} p_{2}}{2 \pi} \frac{d^{2} p_{3}}{2 \pi} \Psi_{\lambda}^{\left(\lambda_{1}, \lambda_{2}\right) \lambda_{3}}\left(\left\{\alpha_{i}\right\},\left\{\boldsymbol{p}_{i}\right\}, \boldsymbol{P}\right) \exp \left(i \sum_{i=1}^{3} \boldsymbol{p}_{i} \cdot \boldsymbol{r}_{i}\right) .
$$

The result takes a rather simple form:

$$
\begin{aligned}
& \tilde{\Psi}_{\lambda}^{\left(\lambda_{1}, \lambda_{2}\right) \lambda_{3}}\left(\left\{\alpha_{i}\right\},\left\{\boldsymbol{r}_{i}\right\}, \boldsymbol{P}\right)=\tilde{\mathcal{N}} \alpha_{1} \alpha_{2} \alpha_{3} \exp \left[-\frac{M^{2}}{4} \sum_{i} \alpha_{i}\left(\boldsymbol{r}_{i}-\boldsymbol{R}\right)^{2}\right] \exp (i \boldsymbol{P} \cdot \boldsymbol{R}) \times \\
& \times\left\{\lambda M \delta_{\lambda, \lambda_{1}} \delta_{\lambda,-\lambda_{2}} \delta_{\lambda, \lambda_{3}}\left[\left(\boldsymbol{r}_{2}-\boldsymbol{R}\right) \cdot \boldsymbol{\eta}_{\lambda}\right]\left[\left(\boldsymbol{r}_{1}-\boldsymbol{r}_{3}\right) \cdot \boldsymbol{\eta}_{-\lambda}\right]\right. \\
& +\quad \lambda M \delta_{\lambda,-\lambda_{1}} \delta_{\lambda, \lambda_{2}} \delta_{\lambda, \lambda_{3}}\left[\left(\boldsymbol{r}_{1}-\boldsymbol{R}\right) \cdot \boldsymbol{\eta}_{\lambda}\right]\left[\left(\boldsymbol{r}_{2}-\boldsymbol{r}_{3}\right) \cdot \boldsymbol{\eta}_{-\lambda}\right]+ \\
& -\quad 2 i \delta_{\lambda, \lambda_{1}} \delta_{\lambda,-\lambda_{2}} \delta_{\lambda,-\lambda_{3}}\left[\left(\boldsymbol{r}_{2}-\boldsymbol{r}_{3}\right) \cdot \boldsymbol{\eta}_{\lambda}\right]+ \\
& \left.-\quad 2 i \delta_{\lambda,-\lambda_{1}} \delta_{\lambda, \lambda_{2}} \delta_{\lambda,-\lambda_{3}}\left[\left(\boldsymbol{r}_{1}-\boldsymbol{r}_{3}\right) \cdot \boldsymbol{\eta}_{\lambda}\right]\right\}
\end{aligned}
$$

where $\boldsymbol{R}$ denotes the light-cone center of mass position vector,

$$
\boldsymbol{R}=\sum_{i=1}^{3} \alpha_{i} \boldsymbol{r}_{i}
$$

The form of the wave function given by Eq. (93) which follows from the Ioffe current shows in detail the angular momentum structure of the baryon and the correlations between the angular momenta and quark helicities. In particular, each scalar product of the type $\left(\boldsymbol{r}_{1}-\boldsymbol{R}\right) \cdot \boldsymbol{\eta}_{\lambda}$ clearly indicates a rotation of quark 1 around the baryon center-of-mass with the orbital angular momentum $z$-component, $L_{z}$, equal to $\lambda$. Terms of the type $\left(\boldsymbol{r}_{1}-\boldsymbol{r}_{3}\right) \cdot \boldsymbol{\eta}_{\lambda}$ correspond to a similar rotation within the quark pair $(1,3)$. Thus, in the massless quark case, all components of the baryon carry a non-zero angular momentum $L_{z}$ for the Ioffe operator. An inspection of the momentum space expressions (32) shows that for the massive quark case, one may have Ioffe baryon wave function components with $L_{z}=0$.

Using Eqs. (43) and (39), one may express the baryon impact factors $\mathcal{B}_{N}^{\lambda \lambda^{\prime}}\left(\left\{\boldsymbol{l}_{i}\right\} ; \boldsymbol{P}, \boldsymbol{P}^{\prime}\right)$ via the overlap function $\mathcal{F}^{\lambda \lambda^{\prime}}\left(\left\{\boldsymbol{l}_{i}\right\} ; \boldsymbol{P}, \boldsymbol{P}^{\prime}\right)$ defined in the coordinate space:

$$
\begin{gathered}
\mathcal{F}^{\lambda \lambda^{\prime}}\left(\left\{\boldsymbol{l}_{i}\right\} ; \boldsymbol{P}, \boldsymbol{P}^{\prime}\right)= \\
\sum_{\lambda_{1}, \lambda_{2}, \lambda_{3}} \int\left[d^{2} \boldsymbol{r}_{i}\right]\left[d \alpha_{i}\right]\left[\tilde{\Psi}_{\lambda^{\prime}}^{\left(\lambda_{1}, \lambda_{2}\right) \lambda_{3}}\left(\left\{\alpha_{i}\right\},\left\{\boldsymbol{r}_{i}\right\} ; \boldsymbol{P}^{\prime}\right)\right]^{*} \exp \left(-i \sum_{i=1}^{3} \boldsymbol{l}_{i} \cdot \boldsymbol{r}_{i}\right) \tilde{\Psi}_{\lambda}^{\left(\lambda_{1}, \lambda_{2}\right) \lambda_{3}}\left(\left\{\alpha_{i}\right\},\left\{\boldsymbol{r}_{i}\right\} ; \boldsymbol{P}\right) .
\end{gathered}
$$

It follows from Eqs. (45), (46) and (95) that the normalization condition for the wave function reads:

$$
\sum_{\lambda_{1}, \lambda_{2}, \lambda_{3}} \int\left[d^{2} \boldsymbol{r}_{i}\right]\left[d \alpha_{i}\right] \quad\left[\tilde{\Psi}_{\lambda}^{\left(\lambda_{1}, \lambda_{2}\right) \lambda_{3}}\left(\left\{\alpha_{i}\right\},\left\{\boldsymbol{r}_{i}\right\} ; \boldsymbol{P}^{\prime}\right)\right]^{*} \tilde{\Psi}_{\lambda}^{\left(\lambda_{1}, \lambda_{2}\right) \lambda_{3}}\left(\left\{\alpha_{i}\right\},\left\{\boldsymbol{r}_{i}\right\} ; \boldsymbol{P}\right)=\delta^{(2)}\left(\boldsymbol{P}-\boldsymbol{P}^{\prime}\right) .
$$


It is instructive to evaluate a contribution to the baryon two-gluon impact factor $\left[\delta \mathcal{B}_{2 ; 0}\right]^{\{1,2\}}$ corresponding to a dipole-like piece, e.g. to $D_{2 ; 0}^{\{1,2\}}$, in the coordinate representation. The gluon color labels are $a_{1}$ and $a_{2}$ and momenta are denoted by $\boldsymbol{k}_{1}$ and $\boldsymbol{k}_{2}$ respectively. One obtains:

$$
\begin{gathered}
{\left[\delta \mathcal{B}_{2 ; 0}^{\lambda \lambda^{\prime}}\left(\left\{\boldsymbol{l}_{i}\right\} ; \boldsymbol{P}, \boldsymbol{P}^{\prime}\right)\right]^{\{1,2\}}=\frac{1}{2}(-i g)^{2} \frac{\delta^{a_{1} a_{2}}}{2 N_{c}} \times} \\
\times \sum_{\lambda_{1}, \lambda_{2}, \lambda_{3}} \int\left[d^{2} \boldsymbol{r}_{i}\right]\left[d \alpha_{i}\right]\left[\tilde{\Psi}_{\lambda^{\prime}}^{\left(\lambda_{1}, \lambda_{2}\right) \lambda_{3}}\left(\left\{\alpha_{i}\right\},\left\{\boldsymbol{r}_{i}\right\} ; \boldsymbol{P}^{\prime}\right)\right]^{*} \tilde{\Psi}_{\lambda}^{\left(\lambda_{1}, \lambda_{2}\right) \lambda_{3}}\left(\left\{\alpha_{i}\right\},\left\{\boldsymbol{r}_{i}\right\} ; \boldsymbol{P}\right) \times \\
\times\left[e^{-i\left(\boldsymbol{k}_{1}+\boldsymbol{k}_{2}\right) \cdot \boldsymbol{r}_{1}}+e^{-i\left(\boldsymbol{k}_{1}+\boldsymbol{k}_{2}\right) \cdot \boldsymbol{r}_{2}}-e^{-i \boldsymbol{k}_{1} \cdot \boldsymbol{r}_{1}-i \boldsymbol{k}_{2} \cdot \boldsymbol{r}_{2}}-e^{-i \boldsymbol{k}_{1} \cdot \boldsymbol{r}_{2}-i \boldsymbol{k}_{2} \cdot \boldsymbol{r}_{1}}\right]
\end{gathered}
$$

Assuming, for simplicity, the forward kinematics, $\boldsymbol{k}_{1}=\boldsymbol{k}=-\boldsymbol{k}_{2}$, one may rewrite the eikonal factors in the last line of (97) in a factorized form, found in the case of the color dipole scattering,

$$
\left[1-e^{i \boldsymbol{k} \cdot\left(\boldsymbol{r}_{2}-\boldsymbol{r}_{1}\right)}\right]\left[1-e^{i \boldsymbol{k} \cdot\left(\boldsymbol{r}_{2}-\boldsymbol{r}_{1}\right)}\right]^{*} .
$$

This equivalence of the structures holds also beyond the forward limit (note that, for nonzero $\boldsymbol{P}, \boldsymbol{P}^{\prime}$ the wave functions $\tilde{\Psi}_{\lambda^{\prime}}^{\left(\lambda_{1}, \lambda_{2}\right) \lambda_{3}}$ contain the phase factors $\left.e^{i \boldsymbol{P R}}\right)$. In Eq. (97), the prefactor $1 / 2$ in the first line reflects the relative weight between the color dipole scattering amplitude and the scattering amplitude of the dipole-like components of the baryon.

\section{The quark-diquark limit}

In many phenomenological applications the nucleon is represented as a bound state of quark and a tightly bound diquark. The transverse size of the diquark is then assumed to be much smaller than the size of the baryon, and the diquark state emerges in an anti-triplet color representation. In this approximation the baryon should resemble an (asymmetric) color dipole. It is interesting to analyze the properties of our baryon impact factor in this limit. Formally, the quark-diquark limit corresponds to the limit where the transverse separation of two quark lines shrinks to zero, and a $t$-channel gluon no longer distinguishes between the two quark lines. In momentum space, as seen in (95), the overlap function then only depends upon the sum of the momenta of all gluons coupled to the two coinciding quark lines. To be definite, let us assume that quarks 2 and 3 move close to each other. Then all overlap functions $F$ degenerate to a function $F^{1(23)}$ with only two arguments:

$$
F\left(\boldsymbol{k}_{1}, \boldsymbol{k}_{2}, \boldsymbol{k}_{3}\right) \underset{3 \rightarrow 2}{\longrightarrow} F^{1(23)}\left(\boldsymbol{k}_{1}, \boldsymbol{k}_{2}+\boldsymbol{k}_{3}\right)
$$

(note that the limit $F^{1(23)}\left(\boldsymbol{k}_{1}, \boldsymbol{k}_{2}\right)$ is not necessarily symmetric in its arguments). Applying this argument to the three dipole-like terms in (49) we immediately see that the dipole-like component $D_{2 ; 0}^{\{2,3\}}$ vanishes if lines 2 and 3 are contracted: this is the well-known limit of a dipole with vanishing size (color transparency). In more detail, (52) shows that all terms in this impact factor tend to $F^{1(23)}\left(0, \boldsymbol{k}_{1}+\boldsymbol{k}_{2}\right)$, and they cancel due to opposite signs. The remaining dipole-like components $D_{2 ; 0}^{\{1,2\}}$ and $D_{2 ; 0}^{\{1,3\}}$ become equal:

$$
D_{2 ; 0}^{\{1,2\}}\left(\boldsymbol{k}_{1}, \boldsymbol{k}_{2}\right), D_{2 ; 0}^{\{1,3\}}\left(\boldsymbol{k}_{1}, \boldsymbol{k}_{2}\right) \underset{3 \rightarrow 2}{\longrightarrow} D_{2 ; 0}^{\{1,(23)\}}\left(\boldsymbol{k}_{1}, \boldsymbol{k}_{2}\right),
$$


with

$$
D_{2 ; 0}^{\{1,(23)\}}\left(\boldsymbol{k}_{1}, \boldsymbol{k}_{2}\right)=\frac{-g^{2}}{12}\left[F^{1(23)}\left(0, \boldsymbol{k}_{1}+\boldsymbol{k}_{2}\right)+F^{1(23)}\left(\boldsymbol{k}_{1}+\boldsymbol{k}_{2}, 0\right)-F^{1(23)}\left(\boldsymbol{k}_{1}, \boldsymbol{k}_{2}\right)-F^{1(23)}\left(\boldsymbol{k}_{2}, \boldsymbol{k}_{1}\right)\right] .
$$

As we already discussed at the end of Section 4.2, in (49) each dipole-like term carries a color factor $1 / 2$, compared to a genuine color dipole factor seen in a color singlet quark-antiquark system. Since in the quark-diquark limit $D_{2 ; 0}^{\{2,3\}}$ vanishes and the contributions from $D_{2 ; 0}^{\{1,2\}}$ and $D_{2 ; 0}^{\{1,3\}}$ coincide, this part of the baryonic impact factor adds up to a standard dipole contribution $D_{2 ; 0}\left(\boldsymbol{k}_{1}, \boldsymbol{k}_{2}\right)=2 D_{2 ; 0}^{\{1,(23)\}}\left(\boldsymbol{k}_{1}, \boldsymbol{k}_{2}\right)$.

Next, we turn to the three-gluon impact factors. In the Pomeron channel, one finds only reggeizing pieces of the quark-diquark dipole impact factor. In the odderon channel, the function $E\left(\boldsymbol{k}_{1}, \boldsymbol{k}_{2}, \boldsymbol{k}_{3}\right)$ degenerates to the structure found in the $\gamma^{*} \rightarrow \eta_{c}$ transition impact factor, which couples only to the Bartels-Lipatov-Vacca (BLV) odderon [33] but not to the Janik-Wosiek solution [34]:

$$
E_{3 ; 0}\left(\boldsymbol{k}_{1}, \boldsymbol{k}_{2}, \boldsymbol{k}_{3}\right) \underset{3 \rightarrow 2}{\longrightarrow} E_{3 ; 0}^{\{1,(23)\}}\left(\boldsymbol{k}_{1}, \boldsymbol{k}_{2}, \boldsymbol{k}_{3}\right)
$$

with

$$
\begin{gathered}
E_{3 ; 0}^{\{1,(23)\}}\left(\boldsymbol{k}_{1}, \boldsymbol{k}_{2}, \boldsymbol{k}_{3}\right)=\frac{i g^{3}}{12}\left[F^{1(23)}\left(\boldsymbol{k}_{1}, \boldsymbol{k}_{2}+\boldsymbol{k}_{3}\right)-F^{1(23)}\left(\boldsymbol{k}_{2}+\boldsymbol{k}_{3}, \boldsymbol{k}_{1}\right)+\right. \\
+F^{1(23)}\left(\boldsymbol{k}_{2}, \boldsymbol{k}_{1}+\boldsymbol{k}_{3}\right)-F^{1(23)}\left(\boldsymbol{k}_{1}+\boldsymbol{k}_{3}, \boldsymbol{k}_{2}\right)+F^{1(23)}\left(\boldsymbol{k}_{3}, \boldsymbol{k}_{1}+\boldsymbol{k}_{2}\right)-F^{1(23)}\left(\boldsymbol{k}_{1}+\boldsymbol{k}_{2}, \boldsymbol{k}_{3}\right)+ \\
\left.+F^{1(23)}\left(\boldsymbol{k}_{1}+\boldsymbol{k}_{2}+\boldsymbol{k}_{3}, 0\right)-F^{1(23)}\left(0, \boldsymbol{k}_{1}+\boldsymbol{k}_{2}+\boldsymbol{k}_{3}\right)\right]
\end{gathered}
$$

For the four gluon case, one finds the standard reggeizing pattern of $D_{2 ; 0}^{\{1,(23)\}}$ and of $E_{3 ; 0}^{\{1,(23)\}}$ in the Pomeron and the odderon channel, respectively. The structure $Q_{4 ; 0}$ vanishes in the quark-diquark limit. This is the result of a nontrivial cancellation of all three lines of Eq. (66), making use of the identity (69). The pattern given by the impact factors in the small diquark limit is preserved by the small $x$ evolution, in particular $Q_{4}$ vanishes.

In summary, we have verified that, in the quark-diquark limit, the baryon reduces to a dipole-like object with an asymmetric wave function, as it was expected. Conversely, our analysis shows that, outside the diquark limit, the baryon impact factor contains a new piece (related to $Q_{4 ; 0}$ ) which accompanies the appearance of the third dipole-like term, $D_{2 ; 0}^{\{2,3\}}$. A more detailed study of the question, to what extent the baryon wave functions actually favors a diquark state, should start from the Fourier transform of the overlap function, (95), which describes the distribution of the quarks in transverse coordinate space. Further work along these lines is in progress.

\section{Discussion}

In this paper we have investigated the high energy behavior of a baryonic state. We have studied the structure of a baryonic impact factor, its coupling to multi-gluon exchanges and the rapidity evolution of the $t$-channel gluon states. We found it convenient to follow very much the same approach, which has been developed and used for the high energy behavior of a virtual photon (or a heavy quarkonium state). For the scattering of such mesonic states, in the leading logarithmic approximation and in the large- $N_{c}$ limit, 
the high energy behavior allows for the interpretation in terms of color dipoles, and one of the motivations of our investigation was the question to what extent this attractive physical picture can be used also for the scattering of baryonic states.

Compared to the quark-antiquark system created by the photon (or a heavy vector meson), the high energy scattering of baryonic systems consisting of three quarks shows similarities, but also striking differences. First, there is a component of the baryonic impact factor in which two of the three quarks interact with the target whereas the third one acts as a spectator. Here the two-quark subsystem behaves very much in the same way as the color singlet dipole of the quark-antiquark system. In particular, the rapidity evolution is the same as in the case of a virtual photon. This configuration, however, extends beyond the picture of a small "diquark state": we have shown that, in the diquark limit, we recover the dipole picture. But the spectator quark is not necessarily linked (in transverse space) to one of the participating quarks, and our analysis includes also this more general configuration. Second, there is the piece of the baryon impact factor to which the $C$-odd three gluon state (odderon) couples. Third, a new piece of the baryonic impact factor exists which couples to a $C$-even three gluon $t$-channel state, and there is a new vertex which describes the transition from this three gluon state to the four gluon (two Pomeron) state. In the quark-antiquark case, there is no analogue of this contribution.

This third piece may actually be quite essential for the restauration of $s$-channel unitarity in baryon scattering and can therefore not be neglected. Namely, let us consider the scattering of a hypothetical heavy baryon on a large nuclear target; this represents the analogue of the Balitsky-Kovchegov problem for the color dipole scattering. Based on our results, the baryon scattering amplitude $\mathcal{B}$ can be written symbolically as a sum of the following pieces:

$$
\mathcal{B}=\overbrace{\mathcal{D}_{2}^{\{1,2\}}+\mathcal{D}_{2}^{\{1,3\}}+\mathcal{D}_{2}^{\{2,3\}}+\mathcal{Q}_{4}}^{C \text {-even }}+\overbrace{\mathcal{E}_{3}}^{C-\text { odd }} .
$$

Here the first three terms, $\mathcal{D}_{2}^{\{i, j\}}$, stand for the dipole-like contributions in which the baryon couples to the same two-point gluon correlator as the color dipole in the scattering of a virtual photon. The strength of this coupling, however, is only $1 / 2$ of that for the photon dipole. The pieces $\mathcal{Q}_{4}$ and $\mathcal{E}_{3}$ probe three-point gluon correlators: the $C$-even and $C$-odd ones respectively. As it was observed in the case of the color dipole in deep inelastic scattering, where only a single BFKL Pomeron could couple to the dipole, we again see no indications of a direct two Pomeron coupling to the valence degrees of freedom of the baryon. If we assume that the two-gluon distribution probed by the first three terms in Eq. (104) is consistent with saturation of the black disc limit for color dipoles of the sizes given by the baryon geometry, then the $T$-matrices for each of the $\mathcal{D}_{2}^{\{i, j\}}$ components would tend to $1 / 2$, and the total contribution of the dipole-like pieces to the baryon $T$-matrix would amount to $3 / 2$. This would mean that $s$-channel unitarity can be maintained only if $\mathcal{Q}_{4}$ and $\mathcal{E}_{3}$ give a combined contribution to the $T$-matrix smaller than $-1 / 2$. Thus, the three-Reggeon states $\mathcal{Q}_{4}$ and $\mathcal{E}_{3}$ seem to be essential to guarantee the $s$-channel unitarity. Interestingly enough, one might go even further and arrive at a quantitative prediction: if one postulates that the $T$-matrices, both for the color dipole and the baryon scattering at very large energies saturate the unitarity limit — one then finds that in the black disc limit: (i) the $C$-odd three point gluon correlator should vanish; this comes from 
the requirement that both proton and anti-proton scattering should reach the black disc limit, despite the fact that the amplitude $\mathcal{E}_{3}$ has opposite signs in these two cases; (ii) the $C$-even three point correlator is strongly constrained: when coupled to the impact factor $Q_{4 ; 0}$ it must lead to the scattering amplitude equal to $-1 / 2$. In the diquark limit, both $D_{2}^{\{2,3\}}$ and $\mathcal{Q}_{4}$ vanish, and unitarization proceeds in the same way as in the dipole case.

We interpret these results as a strong indication that, in the context of baryon scattering, QCD Reggeon field theory has to be extended beyond the theory of BFKL Pomerons and their interactions. First, it is difficult to justify the large- $N_{c}$ limit, which, in the scattering of virtual photon and mesonic states, allows to reduce the evolution of BKP states consisting of $2 n$-gluon to the propagation of $n$ BFKL Pomerons. Second, the three gluon state (and its BKP evolution) seems to play an important rôle, not only in the odderon channel. As we have pointed out, this phenomenon is closely connected with the existence of the $d$ Reggeon, the even signature partner of the (odd signature) reggeized gluon.

On a deeper level one may speculate that there exists an intimate connection between the number of valence objects in the impact factor in the fundamental $S U\left(N_{c}\right)$ representation and the maximal number of Reggeons in the BKP state which couple to the impact factor. For the quark-antiquark color dipole only the two-Reggeon BFKL Pomeron couples, and for the baryon containing three quarks we have both twoand three-Reggeon states. We may conjecture that the number of the different BKP states that couple to the baryon in $S U\left(N_{c}\right)$ gauge theory is related to the number of Casimir operators of the gauge group. There exist two Casimir operators of the $S U(3)$ gauge group, and QCD Reggeon field theory (whose basic degrees of freedom are the reggeized gluons) exhibits two 'fundamental excitations' which, in the leading-log approximation, are represented by the two-gluon BFKL Pomeron and by the three-gluon odderon state. For a high energy $S U\left(N_{c}\right)$ baryon we expect that the impact factor, consisting of $N_{c}$ quarks in the fundamental representation, would exhibit all the $2,3, \ldots, N_{c}$ gluon states, and it would hint that the number of fundamental glue excitations may be related to the $N_{c}-1$ Casimir operators of $S U\left(N_{c}\right)$. It seems natural that the gauge group invariants should be mapped onto gauge invariant BKP states. The explicit connection, however, has not been yet established.

Turning to more practical and phenomenological applications, in this paper we have considered a baryonic state consisting of three massive quarks being in a proton-like configuration. One can view such a 'heavy baryonium' state as a convenient theoretical laboratory, very much in the same spirit as previous work on high energy QCD has made use of 'heavy onium' states. On the other hand, we feel that our results might also allow for immediate phenomenological applications. In particular, we have proposed a relativistic invariant model of the proton wave function, including the helicity structure and correlations between helicities and quark angular momenta. Both the model itself and the calculational technique applied may be useful in studies of polarized scattering of the proton and of the proton form-factors. Another potential place of interest is the intermediate $t$ region of proton-proton elastic scattering where, in the days of ISR experiments, a very simple three gluon model had a striking phenomenological success [35]. It should also be quite interesting to study other applications of the model in the context of elastic $p p$ and $p \bar{p}$ scattering and exclusive diffraction at RHIC, Tevatron and the LHC. Finally, we would like to view our study as a preparation for a QCD analysis of multiple scattering in $p p$ collision at the LHC. 


\section{Acknowledgments}

We especially acknowledge the help of G.P. Vacca who contributed in the early stage of this work. We thank C. Ewerz, L. Lipatov, and M. Salvadore for interesting discussions, and A. Białas, S. Bondarenko, M. Diehl, Yu. Kovchegov, E. Levin and R. Peschanski for useful comments. We thank the Galileo Galilei Institute in Florence for the support in the initial phase of this research project. L.M. gratefully acknowledges the support of the DFG grant SFB 676 and the grant of the Polish State Committee for Scientific Research No. 1 P03B 02828.

\section{A Appendix}

\section{A.1 Spinorial matrix elements}

The calculations of the baryon wave functions and of the baryon scattering amplitudes are performed using the light-cone formalism summarized in [23].

Thus we employ the spinor basis defined by

$$
\left.\begin{array}{l}
u_{\uparrow}(p) \\
u_{\downarrow}(p)
\end{array}\right\}=\frac{1}{\sqrt{p^{+}}}\left(p^{+}+\hat{\beta} m+\hat{\boldsymbol{\alpha}} \cdot \boldsymbol{p}\right) \times\left\{\begin{array}{c}
\chi(\uparrow) \\
\chi(\downarrow)
\end{array}\right.
$$

and

$$
\left.\begin{array}{l}
v_{\uparrow}(p) \\
v_{\downarrow}(p)
\end{array}\right\}=\frac{1}{\sqrt{p^{+}}}\left(p^{+}-\hat{\beta} m+\hat{\boldsymbol{\alpha}} \cdot \boldsymbol{p}\right) \times\left\{\begin{array}{c}
\chi(\downarrow) \\
\chi(\uparrow),
\end{array}\right.
$$

where

$$
\chi(\uparrow)=\frac{1}{\sqrt{2}}\left(\begin{array}{c}
1 \\
0 \\
1 \\
0
\end{array}\right), \quad \chi(\downarrow)=\frac{1}{\sqrt{2}}\left(\begin{array}{c}
0 \\
1 \\
0 \\
-1
\end{array}\right)
$$

in the Dirac representation, and the Dirac matrices $\hat{\beta}$ and $\hat{\alpha}$ are related to the $\gamma$-matrices through $\hat{\beta}=\gamma^{0}$ and $\hat{\alpha}^{s}=\gamma^{0} \gamma^{s} ; m$ is the mass of a fermion (or an anti-fermion). In the infinite momentum frame, when $p^{+} \rightarrow \infty$ these spinors tend to the helicity eigenstates, $u_{\uparrow \downarrow}(p) \rightarrow u_{ \pm}(p), v_{\uparrow \downarrow}(p) \rightarrow v_{ \pm}(p)$.

In the calculation of the baryon $\rightarrow$ quarks transition amplitudes it is sufficient to employ spinor matrix elements given in the following tables. Note that we consider a general case in which the masses of the 
spinors $u$ (or $v$ ) and $u^{\prime}$ are given by $m$ and $m^{\prime}$, respectively.

\begin{tabular}{c|c|c} 
Matrix element & $\begin{array}{c}\lambda \rightarrow \lambda^{\prime} \\
\uparrow \rightarrow \uparrow \\
\downarrow \rightarrow \downarrow \\
\bar{u}_{\lambda^{\prime}}^{\prime}(p) \ldots u_{\lambda}(q)\end{array}$ & $\begin{array}{c}\lambda \rightarrow \lambda^{\prime} \\
\uparrow \rightarrow \downarrow \\
\downarrow \rightarrow \uparrow\end{array}$ \\
\hline$\frac{\bar{u}^{\prime}(p)}{\sqrt{p^{+}}} \gamma^{+} \frac{u(q)}{\sqrt{q^{+}}}$ & 2 & 0 \\
$\frac{\bar{u}^{\prime}(p)}{\sqrt{p^{+}}} \gamma^{-} \frac{u(q)}{\sqrt{q^{+}}}$ & $\frac{2}{p^{+} q^{+}}\left[\left(\boldsymbol{p} \cdot \boldsymbol{\eta}_{\mp}\right)\left(\boldsymbol{q} \cdot \boldsymbol{\eta}_{ \pm}\right)+m m^{\prime}\right]$ & $\mp \frac{2}{p^{+} q^{+}}\left(m \boldsymbol{p} \cdot \boldsymbol{\eta}_{ \pm}-m^{\prime} \boldsymbol{q} \cdot \boldsymbol{\eta}_{ \pm}\right)$ \\
& $\eta_{ \pm}^{s} \frac{\boldsymbol{p} \cdot \boldsymbol{\eta}_{\mp}}{p^{+}}+\eta_{\mp}^{s} \frac{\boldsymbol{q} \cdot \boldsymbol{\eta}_{ \pm}}{q^{+}}$ & $\pm \eta_{ \pm}^{s}\left(\frac{m^{\prime}}{p^{+}}-\frac{m}{q^{+}}\right)$ \\
\hline
\end{tabular}

\begin{tabular}{|c|c|c|}
\hline $\begin{array}{l}\text { Matrix element } \\
\bar{v}_{\lambda^{\prime}}^{\prime}(p) \ldots u_{\lambda}(q)\end{array}$ & $\begin{aligned} \lambda & \rightarrow \lambda^{\prime} \\
\uparrow & \rightarrow \uparrow \\
\downarrow & \rightarrow \downarrow\end{aligned}$ & $\begin{aligned} \lambda & \rightarrow \lambda^{\prime} \\
\uparrow & \rightarrow \downarrow \\
\downarrow & \rightarrow \uparrow\end{aligned}$ \\
\hline$\frac{\bar{v}^{\prime}(p)}{\sqrt{p^{+}}} \gamma^{+} \frac{u(q)}{\sqrt{q^{+}}}$ & 0 & 2 \\
\hline$\frac{\bar{v}^{\prime}(p)}{\sqrt{p^{+}}} \gamma^{-} \frac{u(q)}{\sqrt{q^{+}}}$ & $\mp \frac{2}{p^{+} q^{+}}\left(m \boldsymbol{p} \cdot \boldsymbol{\eta}_{ \pm}+m^{\prime} \boldsymbol{q} \cdot \boldsymbol{\eta}_{ \pm}\right)$ & $\frac{2}{p^{+} q^{+}}\left[\left(\boldsymbol{p} \cdot \boldsymbol{\eta}_{\mp}\right)\left(\boldsymbol{q} \cdot \boldsymbol{\eta}_{ \pm}\right)-m m^{\prime}\right]$ \\
\hline$\frac{\bar{v}^{\prime}(p)}{\sqrt{p^{+}}} \gamma_{\perp}^{s} \frac{u(q)}{\sqrt{q^{+}}}$ & $\mp \eta_{ \pm}^{s}\left(\frac{m^{\prime}}{p^{+}}+\frac{m}{q^{+}}\right)$ & $\eta_{ \pm}^{s} \frac{\boldsymbol{p} \cdot \boldsymbol{\eta}_{\mp}}{p^{+}}+\eta_{\mp}^{s} \frac{\boldsymbol{q} \cdot \boldsymbol{\eta}_{ \pm}}{q^{+}}$ \\
\hline
\end{tabular}

As an example, we apply the above formulae to evaluate

$$
\begin{aligned}
\frac{\left[\bar{d}_{\lambda_{3}}\left(p_{3}\right) \gamma_{\mu} w_{\lambda}(P)\right] \cdot\left[\bar{u}_{\lambda_{1}}\left(p_{1}\right) \gamma^{\mu} v_{\lambda_{2}}\left(p_{2}\right)\right]}{\sqrt{P^{+} p_{1}^{+} p_{2}^{+} p_{3}^{+}}} & =\frac{1}{2} \frac{\left[\bar{d}_{\lambda}\left(p_{3}\right) \gamma^{+} w_{\lambda}(P)\right] \cdot\left[\bar{u}_{\lambda_{1}}\left(p_{1}\right) \gamma^{-} v_{\lambda_{2}}\left(p_{2}\right)\right]}{\sqrt{P^{+} p_{1}^{+} p_{2}^{+} p_{3}^{+}}} \\
& +\frac{1}{2} \frac{\left[\bar{d}_{\lambda}\left(p_{3}\right) \gamma^{-} w_{\lambda}(P)\right] \cdot\left[\bar{u}_{\lambda_{1}}\left(p_{1}\right) \gamma^{+} v_{\lambda_{2}}\left(p_{2}\right)\right]}{\sqrt{P^{+} p_{1}^{+} p_{2}^{+} p_{3}^{+}}} \\
& -\frac{\left[\bar{d}_{\lambda}\left(p_{3}\right) \gamma_{\perp}^{s} w_{\lambda}(P)\right] \cdot\left[\bar{u}_{\lambda_{1}}\left(p_{1}\right) \gamma_{\perp}^{s} v_{\lambda_{2}}\left(p_{2}\right)\right]}{\sqrt{P^{+} p_{1}^{+} p_{2}^{+} p_{3}^{+}}}
\end{aligned}
$$

for $\lambda=\lambda_{1}=-\lambda_{2}=\lambda_{3}=+1$. The prefactors: $1 / 2,1 / 2$ and -1 on the r.h.s. are the only non-vanishing elements of the covariant metric tensor $g_{\mu \nu}$ in the light-cone coordinates. In the calculations we find it useful to make use of the following identities for transverse complex vectors $\boldsymbol{\eta}_{ \pm}: \boldsymbol{\eta}_{+}^{*}=\boldsymbol{\eta}^{-}, \quad \boldsymbol{\eta}_{ \pm}^{2}=0$, 
$\boldsymbol{\eta}_{ \pm} \cdot \boldsymbol{\eta}_{\mp}=\left|\boldsymbol{\eta}_{ \pm}\right|^{2}=2$. Thus, assuming that the light quark masses vanish, we obtain:

$$
\begin{aligned}
\frac{\left[\bar{d}_{\lambda_{3}}\left(p_{3}\right) \gamma_{\mu} w_{\lambda}(P)\right] \cdot\left[\bar{v}_{\lambda_{2}}\left(p_{2}\right) \gamma^{\mu} u_{\lambda_{1}}\left(p_{1}\right)\right]^{*}}{\sqrt{P^{+} p_{1}^{+} p_{2}^{+} p_{3}^{+}}}= & \frac{2\left[\left(\boldsymbol{p}_{2} \cdot \boldsymbol{\eta}_{-}\right)\left(\boldsymbol{p}_{1} \cdot \boldsymbol{\eta}_{+}\right)\right]^{*}}{p_{1}^{+} p_{2}^{+}}+\frac{2\left(\boldsymbol{p}_{3} \cdot \boldsymbol{\eta}_{-}\right)\left(\boldsymbol{P} \cdot \boldsymbol{\eta}_{+}\right)}{P^{+} p_{3}^{+}} \\
& -\frac{2\left(\boldsymbol{p}_{3} \cdot \boldsymbol{\eta}_{-}\right)\left(\boldsymbol{p}_{2} \cdot \boldsymbol{\eta}_{-}\right)^{*}}{p_{2}^{+} p_{3}^{+}}-\frac{2\left(\boldsymbol{P} \cdot \boldsymbol{\eta}_{+}\right)\left(\boldsymbol{p}_{1} \cdot \boldsymbol{\eta}_{+}\right)^{*}}{P^{+} p_{1}^{+}} \\
= & 2\left[\frac{\boldsymbol{p}_{2} \cdot \boldsymbol{\eta}_{+}}{p_{2}^{+}}-\frac{\boldsymbol{P} \cdot \boldsymbol{\eta}_{+}}{P^{+}}\right]\left[\frac{\boldsymbol{p}_{1} \cdot \boldsymbol{\eta}_{-}}{p_{1}^{+}}-\frac{\boldsymbol{p}_{3} \cdot \boldsymbol{\eta}_{-}}{p_{3}^{+}}\right] .
\end{aligned}
$$

Using an identity ${ }^{3} \bar{d}_{\lambda_{3}}\left(p_{3}\right) \gamma_{5}=\lambda_{3} \bar{d}_{\lambda_{3}}\left(p_{3}\right)$, and relation (10), one obtains one of the matrix elements described by (21). The matrix elements for all remaining choices of helicities can be derived in the same way.

\section{A.2 A reduction formula for spinors in high energy limit}

We shall prove the following identity for massive Dirac spinors:

$$
\bar{u}(p) \hat{q}(\hat{p}+m+\hat{k})=2 p \cdot q \bar{u}(p+k)+\ldots,
$$

which holds, at the leading accuracy in $s \simeq 2 p \cdot q$, in the high energy limit: $s \gg q^{2}, k^{2}, m^{2}, p \cdot k, q \cdot k$ etc., and for $k_{\perp} \gg k^{+}, k^{-}$. This identity is a useful tool for deriving quark scattering amplitudes by multi-gluon couplings in the eikonal approximation. Using the spinor equation of motion, $\bar{u}(p)(\hat{p}-m)=0$, we get

$$
\bar{u}(p) \hat{q}(\hat{p}+m+\hat{k})=\bar{u}(p)(2 p \cdot q+\hat{q} \hat{k}) \simeq s \bar{u}(p)\left(1+\frac{1}{2 s}[\hat{q}, \hat{k}]\right)
$$

where we used the fact that the anticommutator $\{\hat{q}, \hat{k}\}=2 k \cdot q \ll s$. Furthermore, using the light-cone variables, as defined in Sec. 3, we have

$$
[\hat{q}, \hat{k}]=-2 i \hat{\sigma}_{\alpha \beta} q^{\alpha} k^{\beta} \simeq-2 i \hat{\sigma}_{-r} q^{-} k_{\perp}^{r},
$$

where $r$ is the Lorentz index of the transverse coordinates. Thus one obtains

$$
\bar{u}(p) \hat{q}(\hat{p}+m+\hat{k}) \simeq s \bar{u}(p)\left(1-\frac{i \hat{\sigma}_{-r} k_{\perp}^{r}}{p^{+}}\right) .
$$

The matrices $\hat{\sigma}^{\alpha \beta}$ are proportional to the generators of the Lorentz transformations of the Dirac spinors:

$$
\exp \left(-\frac{i}{4} \sigma_{\alpha \beta} \omega^{\alpha \beta}\right) u(p)=u(\Lambda(\omega) p), \quad \bar{u}(p) \exp \left(\frac{i}{4} \sigma_{\alpha \beta} \omega^{\alpha \beta}\right)=\bar{u}(\Lambda(\omega) p),
$$

where

$$
(\Lambda(\omega) p)^{\mu}=[\Lambda(\omega)]_{\nu}^{\mu} p^{\nu}, \quad \Lambda(\omega)=\exp \left(\frac{1}{2} \omega^{\alpha \beta} L_{\alpha \beta}\right),
$$

and the generators of Lorentz transformations in the vector representation read

$$
\left[L_{\alpha \beta}\right]_{\nu}^{\mu}=g_{\alpha}^{\mu} g_{\nu \beta}-g_{\beta}^{\mu} g_{\nu \alpha} .
$$

\footnotetext{
${ }^{3}$ For a non-zero quark mass $m$, the relation holds approximately in the large energy limit, $\bar{d}_{\lambda_{3}}\left(p_{3}\right) \gamma_{5}=\lambda_{3} \bar{d}_{\lambda_{3}}\left(p_{3}\right)+$ $O\left(m / p_{3}^{+}\right)$.
} 
Since the parameter multiplying $\hat{\sigma}_{-r}$ in Eq. (113) is small, $\beta^{r}=k_{\perp}^{r} / p^{+} \ll 1$, one may write

$$
\bar{u}(p)\left(1-i \hat{\sigma}_{-r} \beta^{r}\right)=\bar{u}(p)\left[\exp \left(-i \hat{\sigma}_{0 r} \beta^{r} / 2\right) \exp \left(i \hat{\sigma}_{3 r} \beta^{r} / 2\right)\right]+O\left(\beta^{2}\right),
$$

where we used the identity $\gamma_{-}=\frac{1}{2}\left(\gamma_{0}-\gamma_{3}\right)$. This equation corresponds to two subsequent infinitesimal Lorentz transformations acting on $\bar{u}(p)$ with the parameters $\omega_{1}^{r 0}=-\omega_{1}^{0 r}=\beta^{r}$ and $\omega_{2}^{3 r}=-\omega_{2}^{r 3}=\beta^{r}$ (and all other components $\omega_{1,2}^{\alpha \beta}=0$ ). This is an infinitesimal boost along the transverse direction $\beta$, and an infinitesimal rotation in the plane spanned by the transverse vector $\boldsymbol{\beta}$ around the $z$-axis. Using (114) one sees that, in leading order in $\beta^{r}$, the boost transforms $p$ in the following way $: p^{0} \rightarrow p^{0}, \boldsymbol{p} \rightarrow \boldsymbol{p}+p^{0} \boldsymbol{\beta}, p^{3} \rightarrow p^{3}$, and the rotation acts as: $p^{0} \rightarrow p^{0}, \boldsymbol{p} \rightarrow \boldsymbol{p}+p^{3} \boldsymbol{\beta}, p^{3} \rightarrow p^{3}$. Thus one obtains

$$
\bar{u}(p)\left(1-i \hat{\sigma}_{-r} \beta^{r}\right)=\bar{u}\left(p^{\prime}\right)+O\left(\beta^{2}\right),
$$

with $p^{\prime}=\left(p^{0}, \boldsymbol{p}+\boldsymbol{\beta} p^{+}, p^{3}\right)$. This proves Eq. (110). The equation for multiple eikonal couplings, Eq. (15),

follows immediately from Eq. (110), after all spinor contractions, $\hat{q}\left(\hat{p}-\hat{k}_{1}-\ldots-\hat{k}_{i}+m\right) \hat{q} \simeq 2 p \cdot q \hat{q}$, are executed.

\section{References}

[1] L. N. Lipatov, Sov. J. Nucl. Phys. 23 (1976) 338 [Yad. Fiz. 23 (1976) 642]; E. A. Kuraev, L. N. Lipatov and V. S. Fadin, Sov. Phys. JETP 45 (1977) 199 [Zh. Eksp. Teor. Fiz. 72 (1977) 377]; I. I. Balitsky and L. N. Lipatov, Sov. J. Nucl. Phys. 28 (1978) 822 [Yad. Fiz. 28 (1978) 1597].

[2] L. N. Lipatov, Phys. Rept. 286 (1997) 131.

[3] V. S. Fadin and L. N. Lipatov, Phys. Lett. B 429 (1998) 127; M. Ciafaloni and G. Camici, Phys. Lett. B 430 (1998) 349; V. S. Fadin and R. Fiore, Phys. Lett. B 610 (2005) 61 [Erratum-ibid. B 621 (2005) 61]; V. S. Fadin and R. Fiore, Phys. Rev. D 72 (2005) 014018.

[4] I. Balitsky, Nucl. Phys. B 463 (1996) 99.

[5] Y. V. Kovchegov, Phys. Rev. D 60 (1999) 034008; Phys. Rev. D 61 (2000) 074018.

[6] J. Bartels, L. N. Lipatov and G. P. Vacca, Nucl. Phys. B 706 (2005) 391.

[7] N. N. Nikolaev and B. G. Zakharov, Z. Phys. C 49 (1991) 607; Z. Phys. C 53 (1992) 331.

[8] A. H. Mueller, Nucl. Phys. B 415 (1994) 373.

[9] J. Bartels, Z. Phys. C 60 (1993) 471.

[10] J. Jalilian-Marian, A. Kovner and H. Weigert, Phys. Rev. D 59 (1999) 014015; J. Jalilian-Marian, A. Kovner, A. Leonidov and H. Weigert, Phys. Rev. D 59 (1999) 014014; E. Iancu, A. Leonidov and L. D. McLerran, Nucl. Phys. A 692 (2001) 583; E. Iancu, A. Leonidov and L. D. McLerran, Phys. Lett. B 510 (2001) 133; E. Iancu and L. D. McLerran, Phys. Lett. B 510 (2001) 145; E. Ferreiro, E. Iancu, A. Leonidov and L. McLerran, Nucl. Phys. A 703 (2002) 489. 
[11] M. Praszałowicz and A. Rostworowski, Acta Phys. Polon. B 29 (1998) 745.

[12] B. L. Ioffe, Nucl. Phys. B 188 (1981) 317 [Erratum-ibid. B 191 (1981) 591].

[13] V. M. Braun, A. Lenz and M. Wittmann, Phys. Rev. D 73 (2006) 094019.

[14] J. Bartels and M. Wüsthoff, Z. Phys. C 66 (1995) 157.

[15] J. Bartels and C. Ewerz, JHEP 9909 (1999) 026.

[16] S. Braunewell and C. Ewerz, Nucl. Phys. A 760 (2005) 141.

[17] J. Bartels, Nucl. Phys. B 151, 293 (1979); Nucl. Phys. B 175, 365 (1980).

[18] J. Kwieciński and M. Praszałowicz, Phys. Lett. B 94 (1980) 413.

[19] M. A. Shifman, A. I. Vainshtein and V. I. Zakharov, Nucl. Phys. B 147 (1979) 385.

[20] I. I. Balitsky and L. N. Lipatov, JETP Lett. 30 (1979) 355 [Pisma Zh. Eksp. Teor. Fiz. 30 (1979) 383].

[21] A. Ivanov and R. Kirschner, Eur. Phys. J. C 29 (2003) 353.

[22] V. Braun, R. J. Fries, N. Mahnke and E. Stein, Nucl. Phys. B 589 (2000) 381 [Erratum-ibid. B 607 (2001) 433].

[23] G. P. Lepage and S. J. Brodsky, Phys. Rev. D 22 (1980) 2157.

[24] S. J. Brodsky, T. Huang, and G. P. Lepage, in: Particles and Fields 2, Procs. of the Banff Summer Institute, Banff, Alberta, 1981, Eds. A.Z. Capri and A.N. Kamal (Plenum, New York, 1981) p. 143.

[25] J. Bolz and P. Kroll, Z. Phys. A 356 (1996) 327.

[26] M. Diehl, Phys. Rept. 388 (2003) 41.

[27] Y. V. Kovchegov, Phys. Rev. D 64 (2001) 114016 [Erratum-ibid. D 68 (2003) 039901].

[28] M. Fukugita and J. Kwieciński, Phys. Lett. B 83 (1979) 1.

[29] J. Czyżewski, J. Kwieciński, L. Motyka and M. Sadzikowski, Phys. Lett. B 398 (1997) 400 [Erratumibid. B 411 (1997) 402].

[30] Y. Hatta, E. Iancu, K. Itakura and L. McLerran, Nucl. Phys. A 760 (2005) 172.

[31] M. Salvadore, "Aspects of multipartonic interactions in Small-x QCD", PhD. thesis, Bologna University, 2006; J. Bartels, M. Salvadore and G.P. Vacca, in preparation.

[32] M. A. Braun and G. P. Vacca, Eur. Phys. J. C 6 (1999) 147.

[33] J. Bartels, L. N. Lipatov and G. P. Vacca, Phys. Lett. B 477 (2000) 178. 
[34] J. Wosiek and R. A. Janik, Phys. Rev. Lett. 79 (1997) 2935; R. A. Janik and J. Wosiek, Phys. Rev. Lett. 82 (1999) 1092.

[35] P. V. Landshoff, Phys. Rev. D 10 (1974) 1024. 